\title{
FeatureSelect: a software for feature selection based on machine learning approaches
}

\author{
Yosef Masoudi-Sobhanzadeh, Habib Motieghader and Ali Masoudi-Nejad
}

\begin{abstract}
Background: Feature selection, as a preprocessing stage, is a challenging problem in various sciences such as biology, engineering, computer science, and other fields. For this purpose, some studies have introduced tools and softwares such as WEKA. Meanwhile, these tools or softwares are based on filter methods which have lower performance relative to wrapper methods. In this paper, we address this limitation and introduce a software application called FeatureSelect. In addition to filter methods, FeatureSelect consists of optimisation algorithms and three types of learners. It provides a user-friendly and straightforward method of feature selection for use in any kind of research, and can easily be applied to any type of balanced and unbalanced data based on several score functions like accuracy, sensitivity, specificity, etc.

Results: In addition to our previously introduced optimisation algorithm (WCC), a total of 10 efficient, well-known and recently developed algorithms have been implemented in FeatureSelect. We applied our software to a range of different datasets and evaluated the performance of its algorithms. Acquired results show that the performances of algorithms are varying on different datasets, but WCC, LCA, FOA, and LA are suitable than others in the overall state. The results also show that wrapper methods are better than filter methods.

Conclusions: FeatureSelect is a feature or gene selection software application which is based on wrapper methods. Furthermore, it includes some popular filter methods and generates various comparison diagrams and statistical measurements. It is available from GitHub (https://github.com/LBBSoft/FeatureSelect) and is free open source software under an MIT license.
\end{abstract}

Keywords: Feature selection, Gene selection, Machine learning, Classification, Regression

\section{Background}

Data preprocessing is an essential component of many classification and regression problems. Some data have an identical effect, some have a misleading effect and others have no effect on classification or regression problems, and the selection of an optimal and minimum size for features can therefore be useful [1]. A classification or regression problem will involve a high time complexity and low performance when a large number of features is used, but will have a low time complexity and high performance for a minimum size and the most effective features. The selection of an optimal set of features

\footnotetext{
* Correspondence: amasoudin@ut.ac.ir; http://LBB.ut.ac.ir Laboratory of system Biology and Bioinformatics, Institute of Biochemistry and Biophysics, University of Tehran, Tehran, Iran
}

with which a classifier or a model can achieve its maximum performance is an nondeterministic polynomial (NP) problem [2]. Meta-heuristic and heuristic approaches can be applied to NP problems. Optimisation algorithms, which are a type of meta-heuristic algorithm, are usually more efficient than other meta-heuristic algorithms. After selecting an optimal subset of features, a classifier can properly classify the data, or a regression model can be constructed to estimate the relationships between variables. A classifier or a regression model can be created using three methods [3]: (i) a supervised method, in which a learner is aware of data labels; (ii) an unsupervised method, in which a learner is unaware of data labels and tries to find the relationship between data; and (iii) a semi-supervised method in which labels of some data are determined whereas others are not specified. In this

(c) The Author(s). 2019 Open Access This article is distributed under the terms of the Creative Commons Attribution 4.0 International License (http://creativecommons.org/licenses/by/4.0/), which permits unrestricted use, distribution, and reproduction in any medium, provided you give appropriate credit to the original author(s) and the source, provide a link to the Creative Commons license, and indicate if changes were made. The Creative Commons Public Domain Dedication waiver (http://creativecommons.org/publicdomain/zero/1.0/) applies to the data made available in this article, unless otherwise stated. 
method, a learner is usually trained using the both labeled and unlabeled samples. This paper introduces a software application named FeatureSelect in which three types of learner are available in: 1- SVM: A support vector machine (SVM) is one possible supervised learning method that can be applied to classification and regression problems. The aim of an SVM is to determine a line that divides two groups with the greatest margin of confidence [4]. 2- ANN: Like SVM, an artificial neural network (ANN) is a supervised learner and tries to find relation between inputs and outputs. 3- DT: Decision tree (DT) is one of the other supervised learners which can be employed for machine learning applications. FeatureSelect comprises two steps: (i) it selects an optimal subset of features using optimisation algorithms; and (ii) it uses a learner (SVM, ANN and DT) to create a classification or a regression model. After each run, FeatureSelect calculates the required statistical results for regression and classification problems, including sensitivity, fall-out, precision, convergence and stability diagrams for error, accuracy and classification, standard deviation, confidence interval and many other essential statistical results. FeatureSelect is straightforward to use and can be applied within many different fields.

Feature extraction and selection are two main steps in machine learning applications. In feature extraction, some attributes of the existing data, intended to be informative, are extracted. As an instance, we can point out some biologically related works such as Pse-in-One [5] and ProtrWeb [6] which enable users to acquire some features from biological sequences like DNA, RNA, or protein. However, all of the derived features are not constructive in process of learning a machine. Therefore, feature selection methods which are used in various fields such as drug design, disease classification, image processing, text mining, handwriting recognition, spoken word recognition, social networks, and many others, are essential. We divide related works into five categories: (i) filter-based; (ii) wrapper-based; (iii) embedded-based; (iv) online-based; (v) and hybrid-based. Some of the more recently proposed methods and algorithms based on mentioned categories are described below.

\section{(i) Filter-based}

Because filter methods, which does not use a learning method and only considers the relevance between features, have low time complexity; many of researchers focused on these methods. In one of related works, a filter-based method has been introduced for use in online stream feature selection applications. This method has acceptable stability and scalability, and can also be used in offline feature selection applications. However, filter feature selection methods may ignore certain informative features [7]. In some cases, data are unbalanced; in other words, they are in a state of skewness. Feature selection for linear data types has also been studied, in a work that provides a framework and selects features with maximum relevance and minimum redundancy. This framework has been compared with stateof-the-art algorithms, and has been applied to nonlinear data [8].

\section{(ii) wrapper-based}

These methods evaluate usefulness of selected features using learner's performance [9]. In a separate study, a feature selection method was proposed in which both unbalanced and balanced data can be classified, based on a genetic algorithm. However, it has been proved that other optimisation algorithms can be more efficient than the genetic algorithm [10]. Feature selection methods not only improve the performance of the model but also facilitate the analysis of the results. One study examines the use of SVMs in multiclass problems. This work proposes an iterative method based on a features list combination that ranks the features and examines only features list combination strategies. The results show that a one-by-one strategy is better than the other strategies examined, for real-world datasets [11].

\section{(iii) embedded-based}

Embedded methods select features when a model is made. For example, the methods which select features using decision tree are placed in this category. One of the embedded methods investigates feature selection with regard to the relationships between features and labels and the relationships among features. The method proposed in this study was applied to customer classification data, and the proposed algorithm was trained using deterministic score models such as the Fisher score, the Laplacian score, and two semisupervised algorithms. This method can also be trained using fewer samples, and stochastic algorithms can improve the performance of the algorithm [12]. As mentioned above, feature selection is currently a topic of great research interest in the field of machine learning. The nature of the features and the degree to which they can be distinguished are not considered. The concept has been introduced and examined for benchmark datasets by Liu, et al. This method is appropriate for multimodal data types [13].

\section{(iv) online-based}

These methods select features using online user tips. In a related work, a feature cluster taxonomy feature selection (FCTFS) method has been introduced. The main goal of FCTFS is the selection of features based on a user-guided mode. The accuracy of this method is lower than that of the other methods [14]. In a separate study, 
an online feature selection method based on the dependency on the $\mathrm{k}$ nearest neighbours (k-OFSD) has been proposed, and this is suitable for high-dimensional datasets. The main motivation for the abovementioned work is the selection of features with a higher ability to separate those for which the performance has been examined using unbalanced data [15]. A library of online feature selection (LOFS) has also been developed using the state-of-art algorithms, for use with MATLAB and OCTAVE. Since the performance of LOFS has not been examined for a range of datasets, its performance has not been investigated [16].

\section{(v) Hybrid-based}

These methods are combination of four above categories. For example, some related works use two-step feature selection methods $[17,18]$. In these methods, a number of features are reduced by the first method, and the second method is then used for further reduction [19]. While some works focus on only one of these categories, a hybrid two-step feature selection method, which combines the filter and wrapper methods, has been proposed for multi-word recognition. It is possible to remove the most discriminative features in the filter method, so that this method is solely dependent on the filter stage [20]. DNA microarray datasets usually have a large size and a large number of features, and feature selection can reduce the size of this dataset, allowing a classifier to properly classify the data. For this purpose, a new hybrid algorithm has been suggested that combines the maximisation of mutual information with a genetic algorithm. Although the proposed method increases the accuracy, it appears that other state-of-the-art optimisation algorithms can improve accuracy to a greater extent than the genetic algorithm [21-23]. Defining a framework for the relationship between Bayesian error and mutual information [24], and proposing a discrete optimisation algorithm based on opinion formation [25] are other hybrid methods.

Other recent topics of study include review studies or feature selection in special area. A comprehensive and extensive review of over various relevant works was carried out by researchers. The scope, applications and restrictions of these works were also investigated [26-28]. Some other related works are as below: Unsupervised feature selection methods [29-31], feature selection using a variable number of features [32], connecting data characteristics using feature selection [33-36], a new method for feature selection using feature self-representation and a low-rank representation [36], integrating feature selection algorithms [37], financial distress prediction using feature selection [38], and feature selection based on a Morisita estimator for regression problems [39]. Figure 1 summarizes and describes the above categories in a graphical manner.

FeatureSelect is placed in the filter, wrapper, and hybrid categories. In the wrapper method, FeatureSelect scores a subset of features instead of scoring features

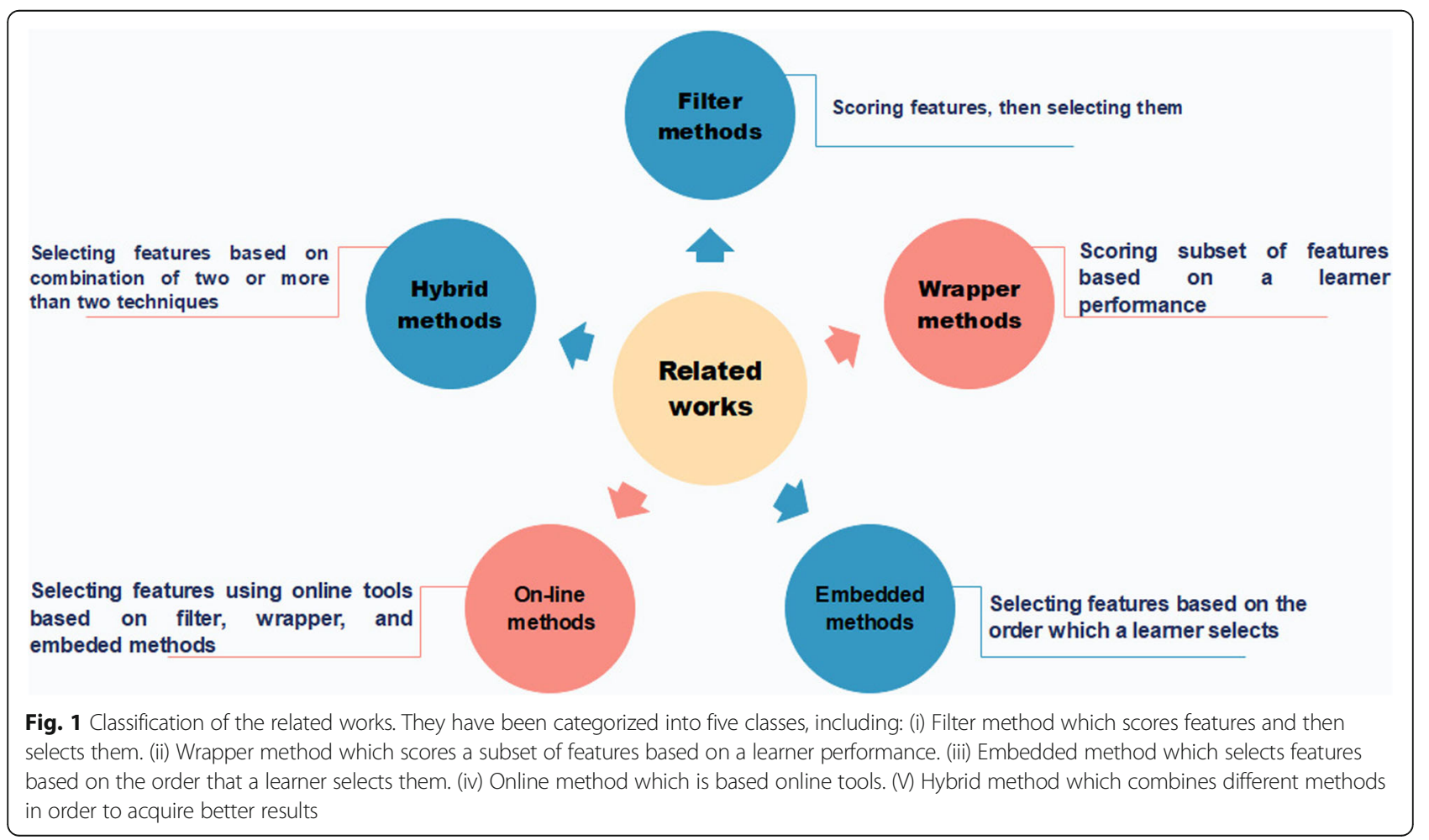


separately. To this end, the optimization algorithms select a subset of features. Next, the selected subset is scored by a learner. In addition to the wrapper method, FeatureSelect includes 5 filter methods which can score features using Laplacian [40], entropy [41], Fisher [42], Pearson-correlation [43], and mutual information [44] scores. After scoring, it selects features based on their scores. Furthermore, this software can be used in a hybrid manner. For example, a user can reduce the number of features using the filter method. Then, the reduced set can be used as input for the wrapper method in order to enhance the performance.

\section{Implementation}

Data classification is a subject that has attracted a great deal of research interest in the domain of machine learning applications. An SVM can be used to construct a hyperplane between groups of data, and this approach can be applied to linear or multiclass classification and regression problems. The hyperplane has a suitable separation ability if it can maintain the largest distance from the points in either class; in other words, the high separation ability of the hyperplane is determined by a functional margin. The higher the value of a functional margin, the lower is the error in the value [45]. Several modified versions of an SVM have also been proposed [46].

Because SVM is a popular classifier in the area of machine learning, Chang and Lin have designed a library for support vector machine named LIBSVM [47], which has several important properties, as follows:

a) It can easily be linked to different programing languages such as MATLAB, Java, Phyton, LISP, CLISP, WEKA, R, C\#, PHP, Haskell, Perl and Ruby;

b) Various SVM formulations and kernels are available;

c) It provides a weighted SVM for unbalanced data;

d) Cross-validation can be applied to the model selection.

In addition to SVM, ANN and DT are also available as learners in FeatureSelect. In the implementation of FeatureSelect, ANN has been implemented whereas SVM and DT have been added to it as a library. ANN, which includes some hidden layers and some neurons in them and can be applied to both classification and regression problems, has been inspired by neural system of living organisms [48]. Like SVM and ANN, DT can also be used for both classification and regression issues. DT operates based on tree-like graph model and develops a tree step by step by adding new constraints which lead to desired consequences [49].

The framework of FeatureSelect is depicted in Fig. 2. The rectangles represent the interaction between FeatureSelect and the user, and the circles represent FeatureSelect processes.

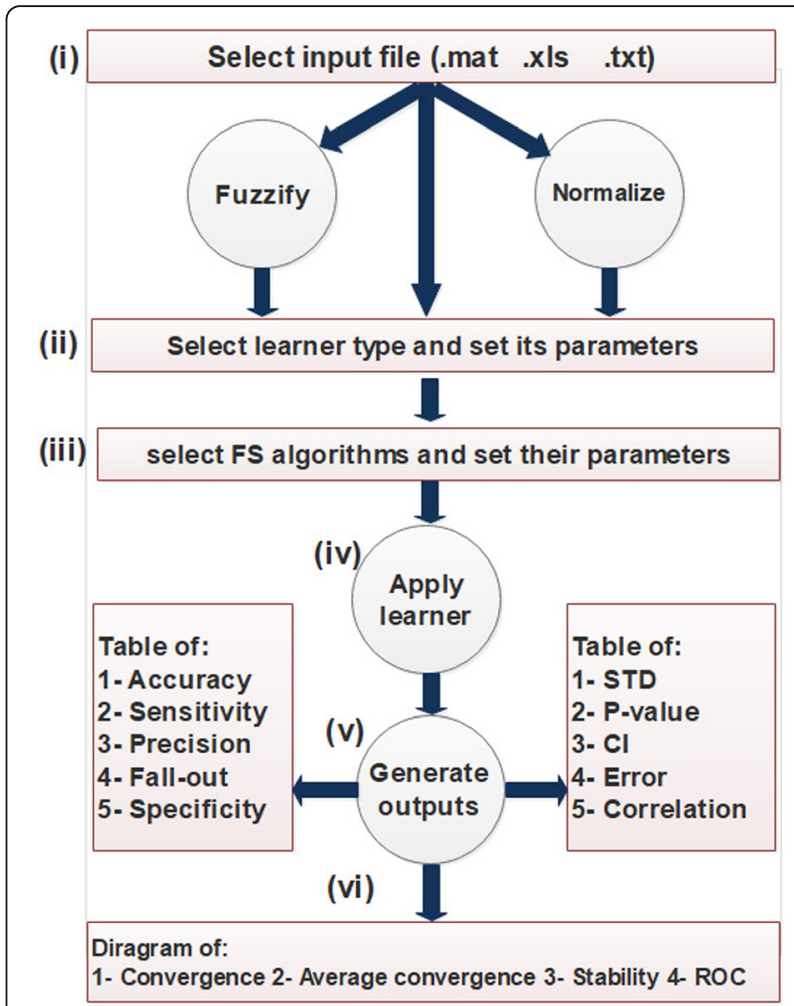

Fig. 2 Framework of FeatureSelect

FeatureSelect consists of six main parts: (i) an input file is selected, and is then fuzzified or normalised if necessary, since this can enhance the learner's functionality; (ii) using a suitable GUI, one of the learners is chosen for classification or regression purpose, and its parameters is adjusted; (iii) one of the two available methods, filter or wrapper method, is selected for feature selection, and then the selected method parameters are determined. In wrapper methods, the list of optimisation algorithms is available. We investigated the performance of 33 optimisation algorithms and have selected 11 state-of-the-art algorithms based on their different natures and performance (Table 1).

(iv) Selected features are evaluated by selected learner. For this purpose, three types of learner can be chosen and adjusted.

(v) FeatureSelect generates various types of results, based on the nature of the problem and selected method, and compares selected algorithms or methods with each other. The status of the executions and selected optimisation algorithms are available in the sixth section.

The relevant properties of FeatureSelect are described below:

a) Data fuzzification and data normalisation capabilities are available. Data are converted to the range $[0,1]$ in both the fuzzification and normalisation stages. TXT, XLS and MAT formats 
Table 1 Implemented algorithms

\begin{tabular}{|c|c|c|c|c|}
\hline Algorithm name & Abrr. & Operations on population & Pub. & Ref \\
\hline $\begin{array}{l}\text { World competitive } \\
\text { contests }\end{array}$ & WCC & $\begin{array}{l}\text { Attacking, shooting, } \\
\text { passing, crossing }\end{array}$ & 2016 & {$[61]$} \\
\hline $\begin{array}{l}\text { League championship } \\
\text { algorithm }\end{array}$ & LCA & Playing, transfer & 2014 & [62] \\
\hline Genetic algorithm & GA & Crossover, mutation & 1970 & [63] \\
\hline $\begin{array}{l}\text { Particle swarm } \\
\text { optimisation }\end{array}$ & PSO & Social behavior & 1995 & [64] \\
\hline Ant colony optimisation & $\mathrm{ACO}$ & $\begin{array}{l}\text { Edge selection, } \\
\text { update pheromone }\end{array}$ & 2006 & {$[65]$} \\
\hline $\begin{array}{l}\text { Imperialist competitive } \\
\text { algorithm }\end{array}$ & ICA & Revolution, absorb, move & 2007 & [66] \\
\hline Learning automata & LA & Award, penalize & 2003 & {$[67]$} \\
\hline $\begin{array}{l}\text { Heat transfer } \\
\text { optimisation }\end{array}$ & HTS & Molecules conductions & 2015 & [68] \\
\hline $\begin{array}{l}\text { Forest optimisation } \\
\text { algorithm }\end{array}$ & $\mathrm{FOA}$ & $\begin{array}{l}\text { Local seeding, } \\
\text { global seeding }\end{array}$ & 2014 & [69] \\
\hline $\begin{array}{l}\text { Discrete symbiotic } \\
\text { organisms search }\end{array}$ & DSOS & $\begin{array}{l}\text { Mutualism, commensalism, } \\
\text { parasitism }\end{array}$ & 2017 & {$[70]$} \\
\hline $\begin{array}{l}\text { Cuckoo optimisation } \\
\text { algorithm }\end{array}$ & CUK & $\begin{array}{l}\text { Eggs laying, eggs killing, } \\
\text { eggs growing }\end{array}$ & 2011 & [71] \\
\hline
\end{tabular}

are acceptable as formats for the input file. Data normalisation is carried out as shown in Eq. 1.

$$
\mathrm{v}^{\prime}=\text { low }+\frac{(v-v \min ) \times(\text { high-low })}{(v \max -v \min )}
$$

where v', v, vmax, vmin, high and low are the normalised value, the current value to be normalised, the maximum and minimum values of the group, and the higher and the lower bounds of the range, respectively. High and low are configured to one and zero respectively in FeatureSelect. Fuzzification is the process that convert scalar values to fuzzy values [50]. Figure 3 illustrates the fuzzy membership function used in FeatureSelect.

b) It provides a suitable graphical user interface for LIBSVM. For example, researchers can select LIBSVM's learning parameters and apply them to their applications after selecting the input data (Fig. 4). If a researcher is unfamiliar with the training and testing functions in LIBSVM, he/she can easily use LIBSVM by clicking on the corresponding buttons.

c) Optimisation algorithms, which are used for feature selection, have been tested and the correctness of them has been examined. Researchers can select one or more of these optimisation algorithms using the relevant box.

d) A user can select different types of learners and feature selection methods, and employee them as ensemble feature selection method. For example, a user can reduce the number of available features by filter methods, and then can use optimisation algorithms or other methods in order to acquire better results.

e) After executing a selected algorithm in a regression problem, FeatureSelect automatically generates useful diagrams and tables, such as the error convergence, error average convergence, error stability, correlation convergence, correlation average convergence and correlation stability diagrams for the selected algorithms in. In classification problems, results include: the accuracy convergence, the accuracy average convergence, the accuracy stability, the error convergence, the error average convergence and the error stability. For both regression and classification problems, an XLS file is generated consisting of a number of selected features, including standard

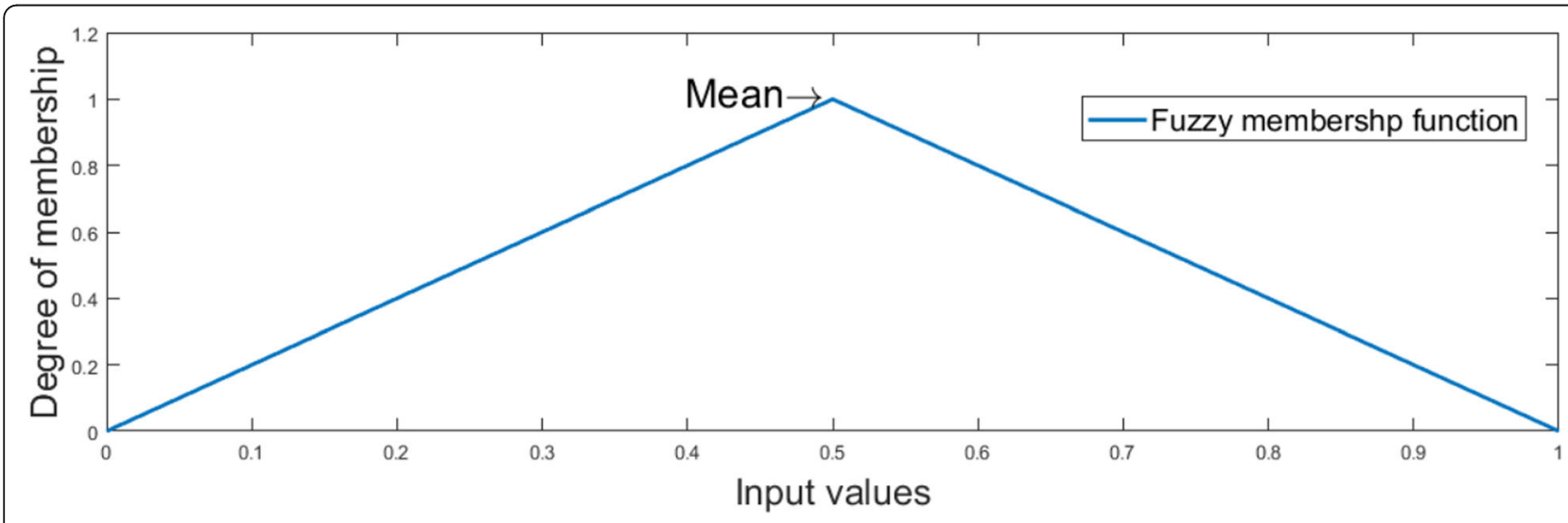

Fig. 3 Fuzzy membership function 


\section{Parameters}

\section{LIB SVM PARAMETERS}

$\square$-s 0 -- C-SVC (multi-class classification)

$\square$-s 1 - nu-SVC (multi-class classification)

$\square$-s 2 -- one-class SVM

$\checkmark$-s 3 -- epsilon-SVR(regression)

$\square$-s 4 -- nu-SVR(regression)

$\square$-t 0 - linear: $u^{* *} \mathrm{v}$

$\square$-t 1 -- polynomial: (gamma* $u^{* *} v+$ coef0 $)^{n}$ degree

$\square$-t 2 -- radial basis function: $\exp (-$ gamma* $|u-v| \wedge 2)$

$\square$-t 3 - sigmoid: $\tanh \left(\right.$ gamma* $u^{* *} v+$ coef0)

$\square$-t 4 -- precomputed kernel (kernel values in training_instance_matrix)

$\square$-d degree : set degree in kernel function 3

$\square$-g gamma : set gamma in kernel function 1

$\square-r$ coef0 : set coef0 in kernel function 0

$\square$-c cost : set the parameter C of C-SVC, epsilon-SVR, and nu-SVR 1

$\square-n$ nu : set the parameter nu of nu-SVC, one-class SVM, and nu-SVR 0.5

$\square$-p epsilon : set the epsilon in loss function of epsilon-SVR 0.1

$\square$-m cachesize : set cache memory size in MB (default 100) 100

$\square$-e epsilon : set tolerance of termination criterion 0.001

$\square$-h shrinking : whether to use the shrinking heuristics, 0 or 1

$\square$-b probability_estimates : whether to train a SVC or SVR model for probability estimates, 0 or 10

$\square$-wi weight : set the parameter $C$ of class i to weight ${ }^{\star} \mathrm{C}$, for $\mathrm{C}$-SVC 1

$\square-v n$ : $n$-fold cross validation mode 5

$\checkmark-q$ : quiet mode (no outputs)

\section{Select and go back}

Fig. 4 Parameters for LIBSVM in FeatureSelect

deviation, $P$-value, confidence interval $(\mathrm{CI})$ and the significance of the generated results, and a TXT file containing detailed information such as the indices of the selected features. For classification problems, certain statistical results such as accuracy, precision, false positive rate, and sensitivity are generated. Eqs. 2 to 5 express how these measures are computed in FeatureSelect, where ACC, PRE, FPR and SEN are abbreviations for accuracy, precision, false positive rate and sensitivity, respectively.

$$
\mathrm{ACC}=\frac{\sum_{i=1}^{n}\left(\frac{T P i+T N i}{T P i+F N i+F P i+T N i}\right) \times C i}{n}
$$

$\mathrm{SEN}=\frac{\sum_{i=1}^{n}\left(\frac{T P i}{T P i+F N i}\right) \times C i}{n}$ 


$$
\begin{aligned}
\mathrm{PRE} & =\frac{\sum_{i=1}^{n}\left(\frac{T P i}{T P i+F P i}\right) \times C i}{n} \\
\mathrm{FPR} & =\frac{\sum_{i=1}^{n}\left(\frac{F P i}{F P i+T N i}\right) \times C i}{n}
\end{aligned}
$$

FeatureSelect obtains results for the average state since it can be applied to both binary and multiple classes of classification problems. In Eqs. 2 to 5, n, TP, TN, FP, FN and $C_{i}$ represent the number of classes, true positive, true negative, false positive, false negative and number of samples in ith class, respectively.

\section{Results}

FeatureSelect has been developed in the MATLAB programming language (Additional file 1), since this is widely used in many research fields such as computer science, biology, medicine and electrical engineering. FeatureSelect can be installed and executed on several operating systems including Windows, Linux and Mac. Moreover, MATLAB-based softwares are open-source, allowing future researchers to add new features to the source code of FeatureSelect.

In this section, we will evaluate the performance of FeatureSelect, and compare its algorithms using various datasets. The eight datasets shown in Table 2 were employed to evaluate the algorithms used in FeatureSelect. Table 2 shows the reference, name, area, number of features (NOF), number of samples (NOS) and number of dataset classes (NOC). Four datasets correspond to classification problems, while the other datasets correspond to regression problems. Using the GitHub link (https://github.com/LBBSoft/FeatureSelect), these datasets can be downloaded.

We ran FeatureSelect on a system with $12 \mathrm{~GB}$ of RAM, a COREi7 CPU and a 64-bit Windows 8.1 operating system. FeatureSelect automatically generates tables and diagrams for selected algorithms and methods. In this paper, we selected all algorithms and compared their operation. Each algorithm was run 30 individual times. Since optimisation algorithms operate randomly, it is advisable to evaluate them over at least 30 individual executions [51]. All the algorithms were run under the same conditions, for example calling an identical number of score functions. Accuracy and root mean squared error (RMSE) [52] were used as the score functions for classification and regression, respectively. The number of generations was set as 50 for all algorithms. We used WCC operators in LCA, since these improve the performance. The datasets (DS) and the name of the algorithm (AL) are shown in the first and second columns of Table 3 (classification datasets) and Table 4 (regression datasets). These tables, in which the best results of each column have been determined, represent certain statistical measures as ready reference for comparing the algorithms. These measures are as follows:

a) NOF: Although the NOF was not applied to score functions, it can be restricted to an upper bound as a maximum number of features or genes in FeatureSelect. The maximum number of features was set as 400, 20, 10, 5, 5, 40, 10, and 5 for the CARCINOMA, BASEHOCK, USPS, DRIVE, AIR, DRUG, SOCIAL, and ENERGY datasets, respectively.

b) Elapsed time (ET): After all algorithms were run 30 times, the best results were selected for each. The ET shows how much time in seconds elapsed in the execution for which the best result was obtained for an algorithm. Algorithms have different ETs due to their various stages.

c) AC: This is a measure that states the rate of correctly predicted samples, relative to all the samples. The difference between AC and ACC is that ACC is an average accuracy for all classes, whereas AC is the accuracy of a specific class. The higher the accuracy, the better the answer.

d) Accuracy standard deviation (AC_STD): This indicates how far the results differ from the mean of the results. It is therefore desirable that AC_STD is a minimum.

\begin{tabular}{|c|c|c|c|c|c|c|}
\hline Name & Type & Area & NOF & NOS & NOC & Ref \\
\hline Social & Regression & Popularity prediction & 59 & 200 & - & {$[72]$} \\
\hline DRUG & Regression & Drug design & 221 & 56 & - & [73] \\
\hline AIR & Regression & Responses to gas multi sensors & 15 & 9358 & - & [74] \\
\hline Energy & Regression & Energy use in low energy building & 29 & 19,735 & - & [75] \\
\hline CARCINOM & Classification & Biology & 9182 & 174 & 11 & [76] \\
\hline USPS & Classification & Hand written image data & 256 & 9298 & 10 & [76] \\
\hline BASEHOCK & Classification & Text data & 1993 & 4862 & 2 & [76] \\
\hline DRIVE & Classification & Driving in real scenario & 606 & 6400 & 3 & [77] \\
\hline
\end{tabular}

Table 2 Datasets 
Table 3 Results obtained for classification datasets using SVM

\begin{tabular}{|c|c|c|c|c|c|c|c|c|c|c|c|c|c|c|c|}
\hline$\overline{D S}$ & $\mathrm{AL}$ & NOF & ET & $A C$ & AC_STD & AC_Cl_L & AC_Cl_H & $A C \_P$ & AC_TS & ER & ER_STD & ER_Cl_L & ER_Cl_H & ER_P & ER_TS \\
\hline \multirow[t]{11}{*}{ (ARCINOM)40\%, N) } & WCC & 319 & 108 & 27.35 & 0.28 & 27.15 & 27.37 & $4.33 \mathrm{E}-69$ & 918.77 & 17.38 & 0.001 & 17.38 & 17.39 & 5.75E-94 & $18,272.5$ \\
\hline & LCA & 270 & 117 & 27.35 & 0.37 & 27.26 & 27.39 & $1.38 \mathrm{E}-65$ & 869 & 17.38 & 0.002 & 17.38 & 17.39 & 1.96E-91 & $13,823.5$ \\
\hline & GA & 487 & 260 & 26.41 & 1.67 & 21.32 & 22.57 & $3.50 \mathrm{E}-34$ & 71.6 & 17.42 & 0.06 & 17.57 & 17.62 & 6.57E-72 & 1435.54 \\
\hline & PSO & 492 & 52 & 27.35 & 2.27 & 25.15 & 26.85 & $1.78 \mathrm{E}-32$ & 62.47 & 17.38 & 0.09 & 17.4 & 17.47 & $6.12 \mathrm{E}-68$ & 1047.51 \\
\hline & $\mathrm{ACO}$ & 491 & 110 & 26.41 & 3.29 & 21.789 & 24.24 & $2.19 \mathrm{E}-26$ & 38.29 & 17.42 & 0.13 & 17.51 & 17.6 & 2.13E-63 & 730.34 \\
\hline & ICA & 488 & 79 & 27.35 & 1.11 & 25.21 & 26.04 & $2.55 \mathrm{E}-41$ & 126.43 & 17.38 & 0.04 & 17.43 & 17.47 & 5.17E-77 & 2152.86 \\
\hline & $\mathrm{LA}$ & 484 & 57 & 26.41 & 6.71 & 15.76 & 20.77 & $3.96 \mathrm{E}-15$ & 14.9 & 17.42 & 0.26 & 17.65 & 17.85 & 1.47E-54 & 361.99 \\
\hline & HTS & 480 & 43 & 26.41 & 3.68 & 18.97 & 21.72 & $1.69 \mathrm{E}-23$ & 30.27 & 17.42 & 0.14 & 17.61 & 17.72 & 4.52E-62 & 657.31 \\
\hline & FOA & 333 & 93 & 28.3 & 0.52 & 27.76 & 28.15 & $7.55 E-52$ & 291.89 & 17.42 & 0.07 & 17.36 & 17.41 & 1.11E-70 & 1301.99 \\
\hline & DSOS & 363 & 78 & 27.35 & 0.23 & 26.38 & 26.56 & 4.79E-61 & 605.92 & 17.38 & 0.009 & 17.41 & 17.42 & $2.58 \mathrm{E}-96$ & 9967.13 \\
\hline & CUK & 408 & 111 & 27.35 & 0.53 & 26.78 & 27.17 & $3.06 \mathrm{E}-51$ & 278.11 & 17.38 & 0.02 & 17.39 & 17.4 & 2.96E-86 & 4484.43 \\
\hline \multirow[t]{11}{*}{$\operatorname{BASEHOCK}(80 \%, \mathrm{O})$} & WCC & 14 & 176 & 72 & 5.33 & 51.03 & 55.01 & $9.17 \mathrm{E}-31$ & 54.48 & 0.18 & 0.05 & 0.45 & 0.49 & 2.93E-29 & 48.28 \\
\hline & LCA & 15 & 140 & 75.25 & 6.57 & 53.91 & 58.82 & $6.49 \mathrm{E}-29$ & 46.96 & 0.25 & 0.07 & 0.41 & 0.46 & $9.64 \mathrm{E}-26$ & 36.35 \\
\hline & GA & 20 & 327 & 48.75 & 0.87 & 46.18 & 46.82 & $6.60 \mathrm{E}-52$ & 293.25 & 0.51 & 0.01 & 0.53 & 0.54 & 1.13E-53 & 337.4 \\
\hline & PSO & 20 & 121 & 50.25 & 1.57 & 45.33 & 46.5 & $2.72 \mathrm{E}-44$ & 160.12 & 0.5 & 0.02 & 0.53 & 0.55 & 2.37E-46 & 188.6 \\
\hline & $\mathrm{ACO}$ & 20 & 140 & 47.75 & 1.1 & 45.01 & 45.83 & 1.09E-48 & 227.11 & 0.52 & 0.01 & 0.54 & 0.55 & $5.28 \mathrm{E}-51$ & 272.95 \\
\hline & ICA & 20 & 165 & 51 & 1.07 & 48.34 & 49.14 & $6.71 \mathrm{E}-50$ & 250.04 & 0.49 & 0.01 & 0.51 & 0.52 & 1.55E-50 & 262.95 \\
\hline & LA & 20 & 81 & 68.25 & 3.8 & 51.1 & 53.94 & $7.28 \mathrm{E}-35$ & 75.61 & 0.32 & 0.04 & 0.46 & 0.49 & $1.33 \mathrm{E}-33$ & 68.36 \\
\hline & HTS & 20 & 65 & 47.5 & 0.89 & 45.32 & 45.98 & $2.25 \mathrm{E}-51$ & 281.07 & 0.53 & 0.01 & 0.54 & 0.55 & 1.43E-53 & 334.63 \\
\hline & $\mathrm{FOA}$ & 16 & 85 & 65.5 & 3.9 & 47 & 49.92 & $1.53 \mathrm{E}-33$ & 68.02 & 0.35 & 0.04 & 0.5 & 0.53 & $2.59 \mathrm{E}-34$ & 72.35 \\
\hline & DSOS & 15 & 118 & 46 & 0.81 & 43.25 & 43.86 & $6.68 \mathrm{E}-52$ & 293.13 & 0.54 & 0.01 & 0.56 & 0.57 & $3.65 E-55$ & 379.83 \\
\hline & CUK & 18 & 138 & 66.25 & 3.04 & 51.37 & 53.64 & $1.16 \mathrm{E}-37$ & 94.51 & 0.34 & 0.03 & 0.46 & 0.49 & 2.10E-36 & 85.48 \\
\hline \multirow[t]{11}{*}{ USPS(80\%, F) } & WCC & 10 & 13 & 85.15 & 0.19 & 84.93 & 85.39 & 4.60E-09 & 290.07 & 2.07 & 0.16 & 1.58 & 1.85 & 0.00001 & 28.5 \\
\hline & LCA & 10 & 12 & 85.15 & 0.83 & 82.93 & 84.99 & 5.27E-09 & 226.64 & 2.15 & 0.26 & 2.06 & 2.7 & 0.00003 & 20.56 \\
\hline & GA & 10 & 10 & 85.15 & 1.5 & 80.71 & 84.44 & $2.62 \mathrm{E}-08$ & 122.97 & 2.56 & 0.38 & 2.1 & 3.05 & 0.00011 & 15.06 \\
\hline & PSO & 10 & 6 & 87.13 & 2.05 & 82.01 & 87.1 & 8.33E-08 & 92.09 & 2.17 & 0.29 & 1.88 & 2.59 & 0.00006 & 17.34 \\
\hline & $\mathrm{ACO}$ & 10 & 17 & 85.15 & 2.03 & 80.85 & 85.89 & 8.41E-08 & 91.87 & 2.91 & 0.48 & 1.57 & 2.77 & 0.00055 & 10.02 \\
\hline & ICA & 10 & 7 & 86.14 & 2.05 & 80.02 & 85.12 & $9.16 \mathrm{E}-08$ & 89.93 & 2.68 & 0.29 & 2.58 & 3.31 & 0.00002 & 22.37 \\
\hline & LA & 10 & 16 & 89.11 & 2.89 & 83.54 & 90.71 & $2.88 \mathrm{E}-07$ & 67.49 & 1.56 & 0.57 & 1.23 & 2.65 & 0.00161 & 7.59 \\
\hline & HTS & 10 & 8 & 81.19 & 1.63 & 77.39 & 81.43 & 4.22E-08 & 109.14 & 3.43 & 0.62 & 3.2 & 4.74 & 0.00013 & 14.33 \\
\hline & $\mathrm{FOA}$ & 10 & 9 & 83.17 & 1.29 & 80.38 & 83.58 & 1.47E-08 & 142 & 1.74 & 0.67 & 1.65 & 3.3 & 0.00113 & 8.33 \\
\hline & DSOS & 10 & 14 & 82.18 & 2.85 & 74.28 & 81.36 & 4.32E-07 & 61.01 & 3.41 & 0.59 & 2.37 & 3.85 & 0.00003 & 11.69 \\
\hline & CUK & 10 & 14 & 84.16 & 1.63 & 80.36 & 84.4 & 3.64E-08 & 113.22 & 2.1 & 0.68 & 1.46 & 3.16 & 0.001637 & 7.56 \\
\hline \multirow[t]{11}{*}{ DRIVE)50\%, N) } & WCC & 3 & 70 & 91.8 & 0.18 & 91.5 & 91.51 & $1.81 \mathrm{E}-76$ & 2759 & 0.08 & 0.001 & 0.08 & 0.09 & 1.05E-45 & 185.46 \\
\hline & LCA & 3 & 69 & 91.8 & 0.26 & 91.34 & 91.54 & $1.62 \mathrm{E}-75$ & 1911.5 & 0.08 & 0.002 & 0.08 & 0.09 & 1.09E-45 & 178.97 \\
\hline & GA & 3 & 16 & 91.8 & 0.33 & 90.95 & 91.2 & 1.67E-72 & 1505.2 & 0.08 & 0.002 & 0.09 & 0.09 & $2.93 \mathrm{E}-43$ & 147.51 \\
\hline & PSO & 3 & 6 & 91.26 & 0.88 & 88.63 & 89.29 & $6.05 E-60$ & 555.22 & 0.09 & 0.01 & 0.11 & 0.11 & $1.06 \mathrm{E}-33$ & 68.89 \\
\hline & $\mathrm{ACO}$ & 3 & 34 & 91.26 & 0.93 & 88.65 & 89.34 & $2.93 \mathrm{E}-59$ & 525.82 & 0.09 & 0.01 & 0.11 & 0.11 & $5.69 \mathrm{E}-33$ & 65 \\
\hline & ICA & 3 & 9 & 91.8 & 0.74 & 90.72 & 91.28 & $2.41 \mathrm{E}-62$ & 671.77 & 0.08 & 0.01 & 0.09 & 0.09 & 3.05E-33 & 66.42 \\
\hline & LA & 3 & 18 & 91.26 & 1.26 & 89.04 & 89.98 & $1.92 \mathrm{E}-55$ & 388.32 & 0.09 & 0.01 & 0.1 & 0.11 & $1.58 \mathrm{E}-28$ & 45.52 \\
\hline & HTS & 3 & 26 & 90.71 & 0.65 & 88.55 & 89.04 & $1.24 \mathrm{E}-63$ & 744.03 & 0.09 & 0.01 & 0.11 & 0.11 & $1.41 \mathrm{E}-37$ & 93.86 \\
\hline & $\mathrm{FOA}$ & 2 & 41 & 91.26 & 0.78 & 88.54 & 89.13 & $2.21 \mathrm{E}-61$ & 622.33 & 0.09 & 0.01 & 0.11 & 0.11 & $2.73 E-35$ & 78.22 \\
\hline & DSOS & 3 & 52 & 91.26 & 0.53 & 88.45 & 88.85 & $3.12 E-66$ & 914.72 & 0.09 & 0.01 & 0.11 & 0.12 & 2.35E-40 & 117.09 \\
\hline & CUK & 3 & 67 & 91.8 & 1.3 & 89.33 & 90.3 & $3.66 \mathrm{E}-55$ & 379.77 & 0.08 & 0.01 & 0.1 & 0.11 & 7.78E-28 & 43.05 \\
\hline
\end{tabular}


Table 4 Results obtained for regression datasets using SVM

\begin{tabular}{|c|c|c|c|c|c|c|c|c|c|c|c|c|c|c|c|}
\hline$\overline{D S}$ & $\mathrm{AL}$ & NOF & ET & ER & ER_STD & ER_Cl_1 & ER_Cl_2 & ER_P & ER_TS & $C R$ & CR_STD & CR_Cl_1 & CR_Cl_2 & CR_P & CR_TS \\
\hline \multirow[t]{11}{*}{$\operatorname{AIR}(80 \%, 0)$} & WCC & 5 & 105 & 0.02 & 0.00 & 0.02 & 0.02 & 0 & $5.3 \mathrm{E}+15$ & 0.60 & 0.00 & 0.60 & 0.60 & 0 & $1.0 \mathrm{E}+15$ \\
\hline & LCA & 5 & 164 & 0.02 & 0.00 & 0.02 & 0.02 & 1.0E-70 & 1306 & 0.60 & 0.00 & 0.60 & 0.60 & $1.25 \mathrm{E}-76$ & 2088.68 \\
\hline & GA & 5 & 73 & 0.02 & 0.00 & 0.02 & 0.02 & $1.3 \mathrm{E}-70$ & 1295.2 & 0.60 & 0.01 & 0.59 & 0.60 & $1.08 \mathrm{E}-54$ & 365.92 \\
\hline & PSO & 5 & 39 & 0.02 & 0.00 & 0.02 & 0.02 & $1.9 \mathrm{E}-55$ & 387.94 & 0.60 & 0.02 & 0.58 & 0.60 & $2.18 \mathrm{E}-42$ & 137.64 \\
\hline & $\mathrm{ACO}$ & 5 & 167 & 0.02 & 0.00 & 0.02 & 0.02 & 8.7E-54 & 340.36 & 0.60 & 0.04 & 0.57 & 0.60 & $2.68 \mathrm{E}-35$ & 78.28 \\
\hline & ICA & 5 & 41 & 0.02 & 0.00 & 0.02 & 0.02 & $6.7 \mathrm{E}-61$ & 598.97 & 0.60 & 0.00 & 0.60 & 0.60 & 2.37E-69 & 1171.79 \\
\hline & LA & 5 & 64 & 0.02 & 0.00 & 0.02 & 0.02 & $7.5 \mathrm{E}-60$ & 551.02 & 0.60 & 0.04 & 0.57 & 0.60 & $2.27 \mathrm{E}-34$ & 72.69 \\
\hline & HTS & 4 & 64 & 0.02 & 0.00 & 0.02 & 0.02 & 3.7E-59 & 521.16 & 0.60 & 0.03 & 0.60 & 0.63 & $2.9 \mathrm{E}-39$ & 107.35 \\
\hline & FOA & 5 & 332 & 0.02 & 0.00 & 0.02 & 0.02 & $4.3 \mathrm{E}-62$ & 658.04 & 0.60 & 0.02 & 0.59 & 0.60 & $4.85 E-46$ & 184.01 \\
\hline & DSOS & 5 & 139 & 0.02 & 0.00 & 0.02 & 0.02 & 7.1E-53 & 316.65 & 0.60 & 0.03 & 0.55 & 0.58 & $1.14 \mathrm{E}-37$ & 94.57 \\
\hline & CUK & 5 & 173 & 0.02 & 0.00 & 0.02 & 0.02 & $2.1 \mathrm{E}-68$ & 1086 & 0.60 & 0.00 & 0.60 & 0.60 & 2.6E-74 & 1737.29 \\
\hline \multirow[t]{11}{*}{ DRUG $(80 \%, N)$} & WCC & 32 & 140 & 0.01 & 0.00 & 0.01 & 0.01 & 2.7E-26 & 38.01 & 0.97 & 0.01 & 0.96 & 0.96 & $1.61 \mathrm{E}-65$ & 864.45 \\
\hline & LCA & 23 & 115 & 0.00 & 0.00 & 0.01 & 0.01 & $3.3 \mathrm{E}-25$ & 34.80 & 0.97 & 0.00 & 0.96 & 0.97 & 4.33E-72 & 1456.43 \\
\hline & GA & 38 & 48 & 0.01 & 0.00 & 0.02 & 0.02 & $1.0 \mathrm{E}-31$ & 58.83 & 0.95 & 0.01 & 0.94 & 0.95 & $1.67 \mathrm{E}-56$ & 422.49 \\
\hline & PSO & 36 & 47 & 0.01 & 0.00 & 0.01 & 0.01 & $9.3 \mathrm{E}-24$ & 30.92 & 0.96 & 0.01 & 0.96 & 0.96 & $3.15 E-63$ & 720.56 \\
\hline & $\mathrm{ACO}$ & 36 & 141 & 0.01 & 0.00 & 0.02 & 0.02 & $9.4 \mathrm{E}-24$ & 30.91 & 0.97 & 0.01 & 0.95 & 0.96 & $1.16 \mathrm{E}-55$ & 395.13 \\
\hline & ICA & 35 & 38 & 0.01 & 0.00 & 0.02 & 0.02 & $6.7 \mathrm{E}-30$ & 50.81 & 0.96 & 0.01 & 0.95 & 0.96 & $5.35 \mathrm{E}-61$ & 603.64 \\
\hline & LA & 30 & 95 & 0.00 & 0.00 & 0.00 & 0.00 & $4.1 \mathrm{E}-24$ & 31.84 & 0.98 & 0.00 & 0.97 & 0.97 & $3.35 E-71$ & 1357.20 \\
\hline & HTS & 32 & 98 & 0.01 & 0.00 & 0.02 & 0.03 & $3.8 \mathrm{E}-25$ & 34.63 & 0.95 & 0.01 & 0.94 & 0.95 & $4.88 \mathrm{E}-57$ & 440.77 \\
\hline & FOA & 20 & 99 & 0.00 & 0.00 & 0.01 & 0.01 & $1.9 \mathrm{E}-18$ & 19.88 & 0.97 & 0.01 & 0.96 & 0.96 & $6.19 \mathrm{E}-66$ & 893.35 \\
\hline & DSOS & 18 & 119 & 0.01 & 0.00 & 0.02 & 0.02 & $7.1 \mathrm{E}-29$ & 46.80 & 0.96 & 0.01 & 0.95 & 0.96 & $3.24 \mathrm{E}-63$ & 719.88 \\
\hline & CUK & 24 & 152 & 0.01 & 0.00 & 0.01 & 0.01 & $1.8 \mathrm{E}-30$ & 53.15 & 0.97 & 0.01 & 0.96 & 0.97 & $4.68 \mathrm{E}-65$ & 833.19 \\
\hline \multirow[t]{11}{*}{ SOCIAL $(80 \%, F)$} & WCC & 8 & 121 & 0.02 & 0.00 & 0.01 & 0.02 & 3.44E-08 & 229.53 & 0.51 & 0.07 & 0.30 & 0.64 & 0.006725 & 12.13 \\
\hline & LCA & 8 & 135 & 0.02 & 0.00 & 0.01 & 0.02 & 4.66E-05 & 146.54 & 0.54 & 0.02 & 0.48 & 0.56 & 0.00033 & 55.01 \\
\hline & GA & 10 & 68 & 0.02 & 0.00 & 0.02 & 0.02 & 0.000558 & 42.33 & 0.36 & 0.04 & 0.23 & 0.44 & 0.005372 & 13.59 \\
\hline & PSO & 10 & 91 & 0.02 & 0.00 & 0.02 & 0.02 & 8.69E-05 & 107.26 & 0.39 & 0.05 & 0.24 & 0.47 & 0.00549 & 13.44 \\
\hline & $\mathrm{ACO}$ & 10 & 153 & 0.02 & 0.00 & 0.02 & 0.02 & 0.000394 & 50.35 & 0.31 & 0.05 & 0.17 & 0.42 & 0.010204 & 9.82 \\
\hline & ICA & 9 & 76 & 0.02 & 0.00 & 0.02 & 0.02 & 0.00017 & 76.61 & 0.37 & 0.01 & 0.36 & 0.39 & $6.79 \mathrm{E}-05$ & 121.39 \\
\hline & LA & 10 & 93 & 0.02 & 0.00 & 0.01 & 0.02 & 0.000485 & 45.39 & 0.53 & 0.02 & 0.45 & 0.57 & 0.000754 & 36.40 \\
\hline & HTS & 8 & 93 & 0.02 & 0.00 & 0.02 & 0.02 & $6.75 E-05$ & 121.73 & 0.36 & 0.03 & 0.23 & 0.41 & 0.003921 & 15.92 \\
\hline & FOA & 8 & 86 & 0.02 & 0.00 & 0.01 & 0.03 & 0.010557 & 9.66 & 0.45 & 0.16 & 0.10 & 0.70 & 0.083971 & 3.23 \\
\hline & DSOS & 8 & 122 & 0.02 & 0.00 & 0.02 & 0.03 & 0.001028 & 31.17 & 0.25 & 0.04 & 0.11 & 0.31 & 0.012132 & 9.00 \\
\hline & CUK & 8 & 93 & 0.02 & 0.00 & 0.02 & 0.02 & 0.000439 & 47.70 & 0.35 & 0.03 & 0.26 & 0.39 & 0.002276 & 20.93 \\
\hline \multirow[t]{11}{*}{ ENERGY $(60 \%, O)$} & WCC & 5 & 64 & 0.08 & 0.00 & 0.08 & 0.08 & $6.03 E-80$ & 2717.4 & 0.5 & 0 & 0.4 & 0.4 & 1.19E-35 & 80.49 \\
\hline & LCA & 5 & 82 & 0.08 & 0.00 & 0.08 & 0.08 & 1.60E-83 & 3609.2 & 0.5 & 0 & 0.4 & 0.4 & $6.82 \mathrm{E}-33$ & 64.59 \\
\hline & $\mathrm{GA}$ & 5 & 23 & 0.08 & 0.00 & 0.08 & 0.08 & 2.70E-75 & 1878.2 & 0.4 & 0 & 0.3 & 0.4 & $3.46 \mathrm{E}-29$ & 48 \\
\hline & PSO & 5 & 25 & 0.08 & 0.00 & 0.08 & 0.08 & $7.82 \mathrm{E}-70$ & 1217.4 & 0.3 & 0.1 & 0.3 & 0.3 & $3.16 \mathrm{E}-23$ & 29.61 \\
\hline & $\mathrm{ACO}$ & 5 & 52 & 0.08 & 0.00 & 0.08 & 0.08 & $1.34 \mathrm{E}-63$ & 742.04 & 0.4 & 0.1 & 0.2 & 0.3 & $1.54 \mathrm{E}-17$ & 18.4 \\
\hline & ICA & 5 & 57 & 0.08 & 0.00 & 0.08 & 0.08 & 4.89E-79 & 2528.3 & 0.5 & 0 & 0.4 & 0.4 & $1.55 E-31$ & 57.95 \\
\hline & LA & 5 & 24 & 0.08 & 0.00 & 0.08 & 0.08 & 1.57E-73 & 1632.7 & 0.5 & 0 & 0.4 & 0.4 & 1.07E-29 & 49.99 \\
\hline & HTS & 4 & 27 & 0.08 & 0.00 & 0.08 & 0.08 & $1.08 \mathrm{E}-66$ & 948.73 & 0.4 & 0.1 & 0.3 & 0.3 & $1.78 \mathrm{E}-18$ & 19.94 \\
\hline & FOA & 5 & 30 & 0.08 & 0.00 & 0.08 & 0.08 & $2.20 \mathrm{E}-66$ & 925.79 & 0.5 & 0.1 & 0.3 & 0.3 & 1.97E-20 & 23.51 \\
\hline & DSOS & 5 & 42 & 0.08 & 0.00 & 0.08 & 0.08 & $3.70 \mathrm{E}-66$ & 909.35 & 0.4 & 0.1 & 0.3 & 0.3 & $6.59 \mathrm{E}-24$ & 31.31 \\
\hline & CUK & 5 & 80 & 0.08 & 0.00 & 0.08 & 0.08 & 2.33E-80 & 2807.9 & 0.5 & 0 & 0.4 & 0.4 & $6.99 \mathrm{E}-32$ & 59.58 \\
\hline
\end{tabular}


e) CI: This represents a range of values, and the results are expected to fall into this range with a maximum specific probability. CI_L and CI_H stand for the lower and higher bounds on the confidence interval.

f) $P$-value of accuracy (AC_P): The $p$-value is a statistical measurement that expresses the extent to which the obtained results are similar to random values. An algorithm with a minimum p-value is more reliable than others.

g) Accuracy test statistic (AC_TS): TS is generally used to reject or accept a null hypothesis. When the TS is a maximum, the $\mathrm{p}$-value is a minimum.

h) Root mean squared error (ER or RMSE): ER is calculated using Eq. 6, where $n, y_{i}$ and $y_{i}^{\prime}$ are the number of samples, and the predicted and label values, respectively. This measurement expresses the average difference between predicted and label values.

$$
\mathrm{ER}=\sqrt{\frac{\left(y i-y^{\prime} i\right)}{n}}
$$

i) Error standard deviation (ER_STD): In the same way as AC_STD, ER_STD indicates how far the RMSE differs from the average RMSE when 30 individual executions are performed. The lower the ER_STD, the closer the obtained results. j) Squared correlation coefficient (CR): The correlation (R) determines the connectivity between the predicted values and label values. CR is calculated based on $R^{2}$. We expect the CR to increase when the error decreases.

The concepts between (ER_CI_L and CR_CI_L and AC_CI_L), between (ER_CI_H and CR_CI_H and AC_CI_H), between (ER_STD and CR_STD and AC_STD), between (AC_P and ER_P and CR_P), and finally between (AC_TS and ER_TS and CR_TS) are alike. In addition to the name of the dataset, the training data percentage and an input data type are specified. Three input data types were used: fuzzified $(\mathrm{F})$, normalised, $(\mathrm{N})$ and ordinary $(\mathrm{O})$.

FeatureSelect generates diagrams for the ACC, average of the ACC and the stability of the ACC for classification datasets. In addition, it generates diagrams of the ER, average ER and stability of the ER for both classification and regression datasets.

The criteria used to evaluate the optimisation algorithms were convergence, average convergence and stability. These measures indicate whether or not the algorithms have been correctly implemented. Figures 5 and 6 illustrate instances of FeatureSelect outputs based on the mentioned criteria. The convergence mean is that the answers must be improved when the number of iterations or time dedicated to the algorithms is increased. For example, we observe that the ER decreases and the $\mathrm{CR}$ and ACC increase with a higher number of iterations. From convergence point of view, all of the algorithms increase the accuracy and correlation, and reduce the error. Although all of them have generated
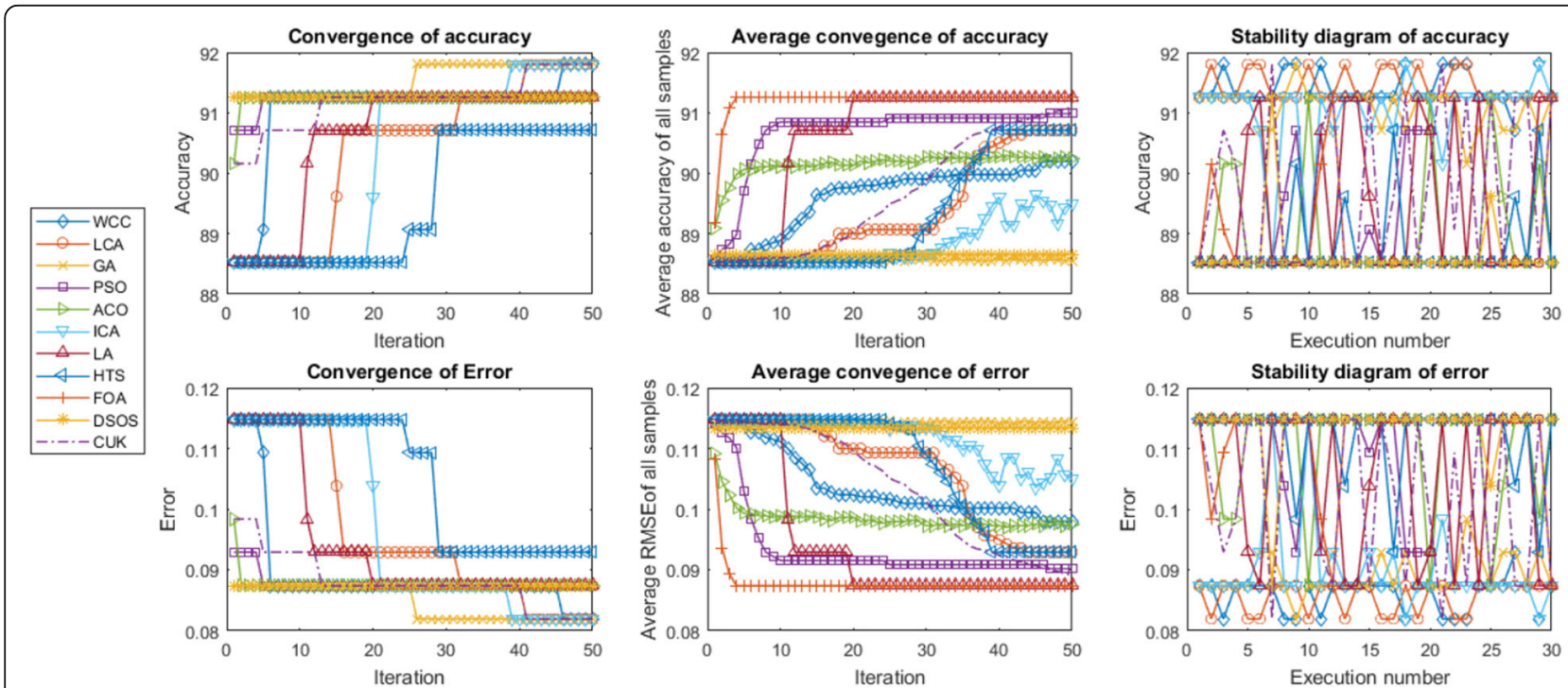

Fig. 5 Diagrams generated for the DRIVE dataset using SVM. These diagrams compare the algorithms performances against each other based on accuracy and error scores. For every score, convergence, average convergence, and stability diagrams have been shown. Given the results on the DRIVE dataset, the performances of WCC, GA, LCA, and LA are better than the others 

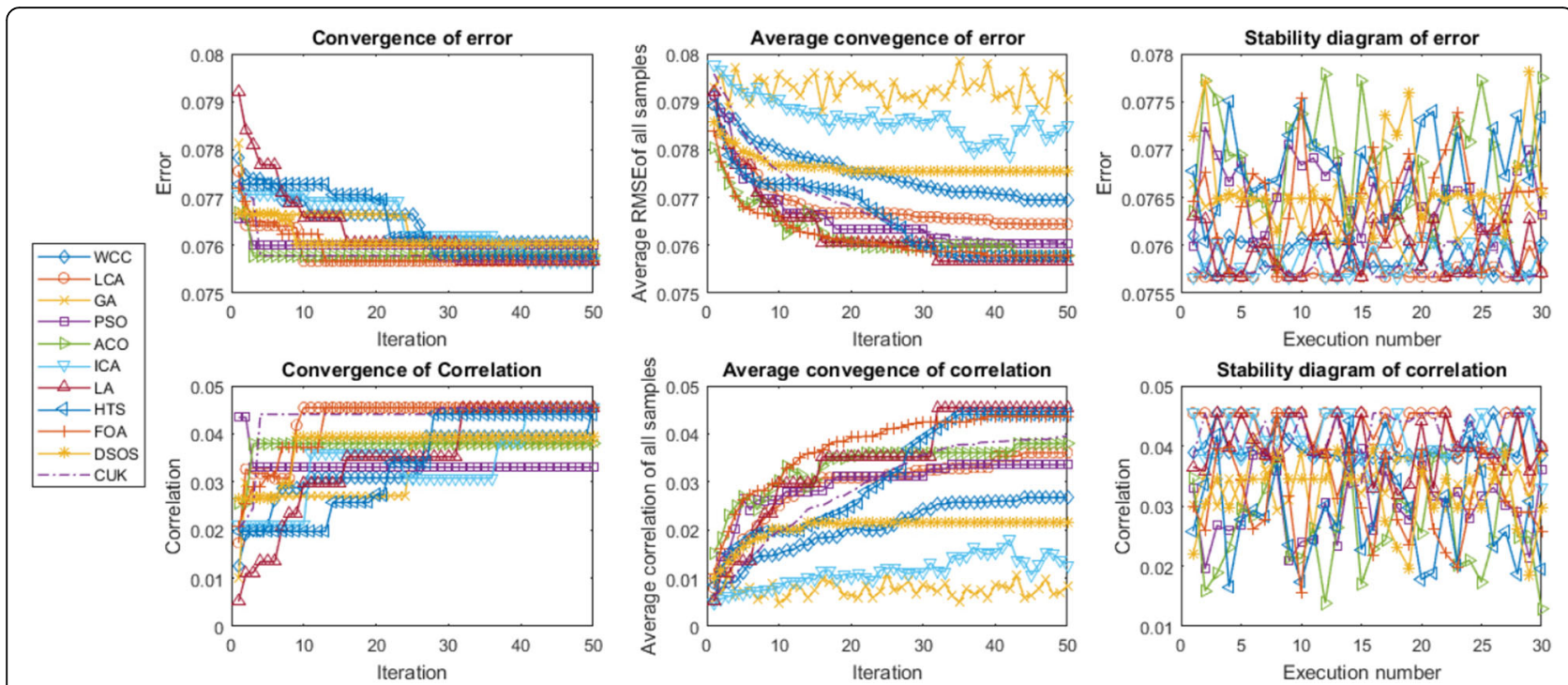

Fig. 6 Diagrams generated for the ENERGY dataset using SVR. These diagrams compare the algorithms performances against each other based on RMSE and correlation scores. For every score, convergence, average convergence, and stability diagrams have been shown. Given the results on the ENERGY dataset, the performances of CUK, HTS, LCA, and LA are proper than the others

acceptable results, LA, LCA, WCC and GA are suitable than others. In addition to convergence, there is the concept of average convergence. The difference between the two is that the convergence is obtained by extracting the best answer at the end of each iteration, whereas average convergence is calculated based on the mean of potential solution scores at the end of each iteration. As it is observable, all of the potential answers generated by algorithms except GA and ICA are improving when the iteration is increased. In order to improve the performance of GA, we replace some of the worst results with randomly created answers at the end of each iteration. Also, absorb operator of ICA makes some countries worse or better than their previous status. Hence, the average convergence of GA and ICA may not have ascending or descending form. Stability diagrams indicate how the results fluctuate from a forward line in the individual executions. An algorithm can be said to be better than others if its results lie on the forward line and if the mean of its results is better than those of other algorithms. The results shown in Tables 3 and 4 have been calculated based on the stability results. FeatureSelect also generates several addition outputs for classification datasets, as follows:

a) Essential statistical measurements: These measures are shown in Eqs. 2 to 5. Table 5 presents these statistical measures for all datasets.

b) Receiver operating characteristic (ROC) curve: This is usually used for binary classification, but has been extended here to multi-class classification. The $\mathrm{ROC}$ is a graphical plot that indicates the diagnostic ability of a classifier. The horizontal axis is FPR (1-specificity) and the vertical axis is TPR (true positive rate or sensitivity) [53]. The ROC curve and ROC space for the algorithms for the USPS dataset are shown in Fig. 7 as an example of FeatureSelect's output for classification datasets.

Like the ROC curve, the ROC space represents the trade-offs between TPR and FPR. A point that is closer to the left and the top represents an algorithm with better diagnostic ability; for example, LCA has the best diagnostic ability for the USPS dataset.

In overall evaluation, we compare the performance of the FeatureSelect algorithms. The values in Tables 6, 7 and 8 are a summary of those in Tables 3,4 and 5 respectively (the average for table), and allow an overall comparison of the algorithms used in FeatureSelect. LCA has selected 74.5 features in the average state on four classification datasets. Although the time orders are the same for all algorithms, the average elapsed time for four classification datasets is 35.5 for HTS. LCA and WCC show similar operation, but the accuracy of LCA is better than that of WCC. Its accuracy confidence interval is also more acceptable than that of the others. We show the AC_P and ER_P using three floating digits.

These values are identical for all algorithms, indicating that the performance of the algorithms is not random. For all classification datasets, FOA reaches a minimum value of ER. Therefore, it is proper than other algorithms in ER point of view. We also observe that WCC operates better than the other algorithms in terms of ER_TS, CR, CR_CI, CR_P and CR_TS. 
Table 5 Essential statistical measurements for all classification datasets

\begin{tabular}{|c|c|c|c|c|c|c|c|c|c|c|c|}
\hline$\overline{\mathrm{DS}}$ & AL_NAME & SEN & PRE & FPR & ACC & DS & AL_NAME & SEN & PRE & FPR & $\mathrm{ACC}$ \\
\hline \multirow[t]{11}{*}{$\overline{\text { CARCINOM }(80 \%, N)}$} & WCC & 0.68 & 0.60 & 0.02 & 0.76 & \multirow[t]{11}{*}{ USPS $(80 \%, 0)$} & WCC & 0.82 & 0.86 & 0.02 & 0.85 \\
\hline & LCA & 0.68 & 0.60 & 0.02 & 0.76 & & LCA & 0.82 & 0.83 & 0.02 & 0.85 \\
\hline & GA & 0.68 & 0.60 & 0.02 & 0.75 & & GA & 0.83 & 0.86 & 0.02 & 0.85 \\
\hline & PSO & 0.68 & 0.60 & 0.02 & 0.76 & & PSO & 0.87 & 0.88 & 0.02 & 0.87 \\
\hline & $\mathrm{ACO}$ & 0.68 & 0.60 & 0.02 & 0.75 & & $\mathrm{ACO}$ & 0.85 & 0.85 & 0.02 & 0.85 \\
\hline & ICA & 0.68 & 0.60 & 0.02 & 0.76 & & ICA & 0.81 & 0.89 & 0.02 & 0.86 \\
\hline & LA & 0.68 & 0.60 & 0.02 & 0.75 & & LA & 0.89 & 0.89 & 0.01 & 0.89 \\
\hline & HTS & 0.68 & 0.60 & 0.02 & 0.58 & & HTS & 0.79 & 0.82 & 0.03 & 0.81 \\
\hline & $\mathrm{FOA}$ & 0.68 & 0.60 & 0.02 & 0.77 & & $\mathrm{FOA}$ & 0.81 & 0.84 & 0.02 & 0.83 \\
\hline & DSOS & 0.68 & 0.60 & 0.02 & 0.76 & & DSOS & 0.80 & 0.80 & 0.02 & 0.82 \\
\hline & CUK & 0.68 & 0.60 & 0.02 & 0.76 & & CUK & 0.82 & 0.84 & 0.02 & 0.84 \\
\hline \multirow[t]{11}{*}{ BASEHOCK $(80 \%, F)$} & WCC & 0.66 & 0.89 & 0.33 & 0.72 & \multirow[t]{11}{*}{ DRIVE $(80 \%, N)$} & WCC & 0.56 & 0.81 & 0.24 & 0.92 \\
\hline & LCA & 0.70 & 0.83 & 0.30 & 0.75 & & LCA & 0.56 & 0.81 & 0.24 & 0.92 \\
\hline & GA & 0.57 & 0.72 & 0.43 & 0.49 & & GA & 0.56 & 0.81 & 0.24 & 0.92 \\
\hline & PSO & 0.58 & 0.71 & 0.42 & 0.50 & & PSO & 0.52 & 0.80 & 0.25 & 0.91 \\
\hline & $\mathrm{ACO}$ & 0.56 & 0.72 & 0.44 & 0.48 & & $\mathrm{ACO}$ & 0.52 & 0.80 & 0.25 & 0.91 \\
\hline & ICA & 0.58 & 0.72 & 0.42 & 0.51 & & ICA & 0.56 & 0.81 & 0.24 & 0.92 \\
\hline & LA & 0.68 & 0.67 & 0.32 & 0.68 & & LA & 0.52 & 0.80 & 0.25 & 0.91 \\
\hline & HTS & 0.53 & 0.71 & 0.47 & 0.44 & & HTS & 0.33 & 0.63 & 0.33 & 0.89 \\
\hline & FOA & 0.58 & 0.75 & 0.42 & 0.66 & & FOA & 0.52 & 0.80 & 0.25 & 0.91 \\
\hline & DSOS & 0.54 & 0.72 & 0.46 & 0.46 & & DSOS & 0.52 & 0.80 & 0.25 & 0.91 \\
\hline & CUK & 0.66 & 0.66 & 0.34 & 0.66 & & CUK & 0.56 & 0.81 & 0.24 & 0.92 \\
\hline
\end{tabular}
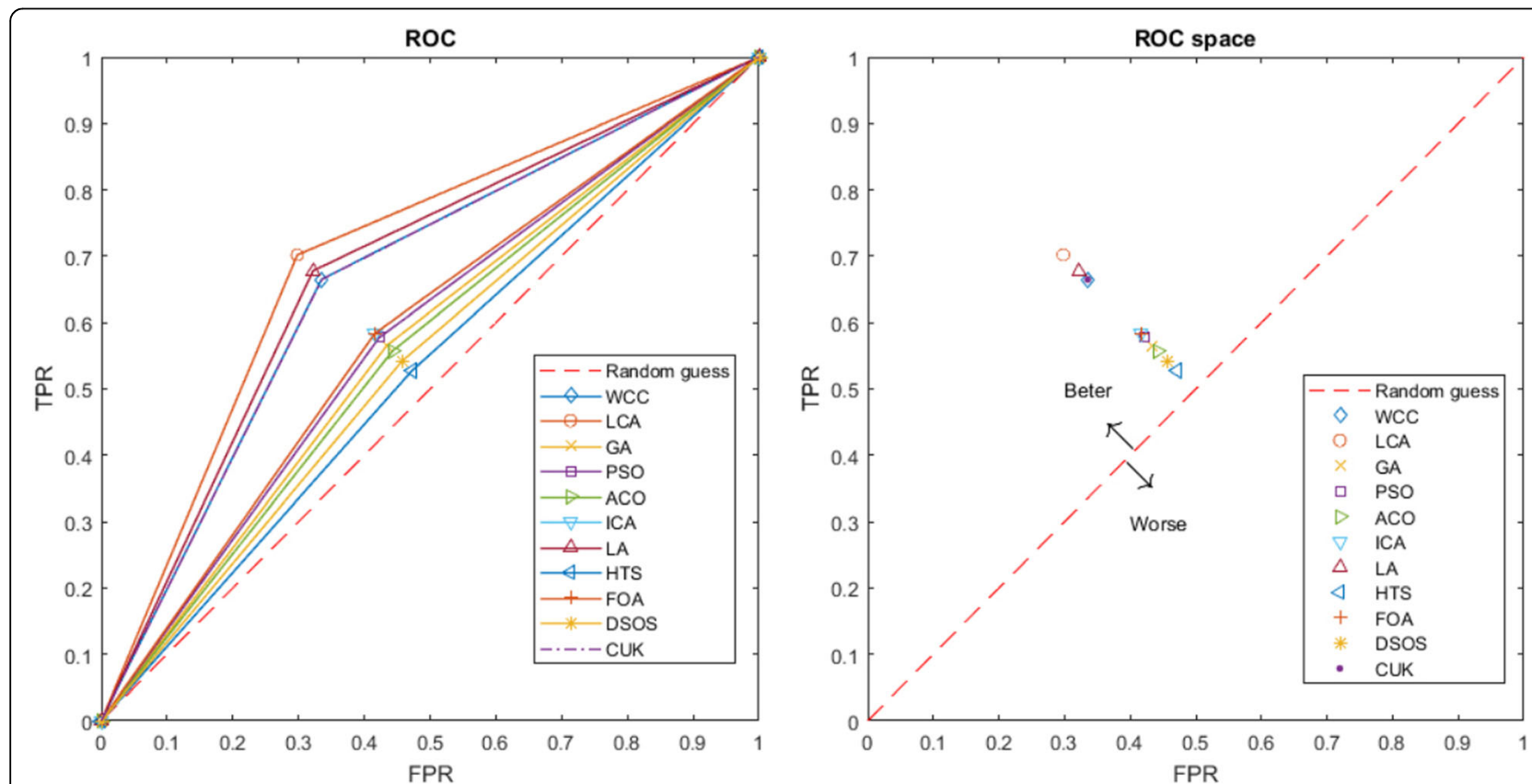

Fig. 7 ROC curve and ROC space for the algorithms used based on SVM 
Table 6 Summary of results for all classification datasets

\begin{tabular}{|c|c|c|c|c|c|c|c|c|c|c|c|c|c|c|}
\hline $\mathrm{AL}$ & NOF & ET & $A C$ & AC_STD & AC_Cl_L & AC_Cl_H & AC_P & AC_TS & ER & ER_STD & ER_Cl_L & ER_Cl_H & ER_P & ER_TS \\
\hline WCC & 86.50 & 91.75 & 69.08 & 1.50 & 63.65 & 64.82 & 0.000 & 1005.58 & 4.93 & 0.05 & 4.94 & 4.96 & 0.000 & 4633.69 \\
\hline LCA & 74.50 & 84.50 & 69.89 & 2.01 & 63.86 & 65.69 & 0.000 & 763.53 & 4.97 & 0.08 & 4.98 & 5.16 & 0.000 & 3514.85 \\
\hline GA & 130.00 & 153.25 & 63.03 & 1.09 & 59.79 & 61.26 & 0.000 & 498.26 & 5.14 & 0.11 & 5.07 & 5.33 & 0.000 & 483.88 \\
\hline PSO & 131.25 & 46.25 & 64.00 & 1.69 & 60.28 & 62.44 & 0.000 & 217.48 & 5.04 & 0.10 & 4.98 & 5.18 & 0.000 & 330.59 \\
\hline ACO & 131.00 & 75.25 & 62.64 & 1.84 & 59.07 & 61.33 & 0.000 & 220.77 & 5.24 & 0.16 & 4.93 & 5.26 & 0.000 & 269.58 \\
\hline ICA & 130.25 & 65.00 & 64.07 & 1.24 & 61.07 & 62.90 & 0.000 & 284.54 & 5.16 & 0.09 & 5.15 & 5.35 & 0.000 & 626.15 \\
\hline LA & 129.25 & 43.00 & 68.76 & 3.67 & 59.86 & 63.85 & 0.000 & 136.58 & 4.85 & 0.22 & 4.86 & 5.28 & 0.000 & 120.87 \\
\hline HTS & 128.25 & 35.50 & 61.45 & 1.71 & 57.56 & 59.54 & 0.000 & 291.13 & 5.37 & 0.20 & 5.37 & 5.78 & 0.000 & 275.03 \\
\hline FOA & 90.25 & 57.00 & 67.06 & 1.62 & 60.92 & 62.70 & 0.000 & 281.06 & 4.90 & 0.20 & 4.91 & 5.34 & 0.000 & 365.22 \\
\hline DSOS & 97.75 & 65.50 & 61.70 & 1.11 & 58.09 & 60.16 & 0.000 & 468.70 & 5.36 & 0.15 & 5.11 & 5.49 & 0.000 & 2618.94 \\
\hline CUK & 109.75 & 82.50 & 67.39 & 1.63 & 61.96 & 63.88 & 0.000 & 216.40 & 4.98 & 0.19 & 4.85 & 5.29 & 0.000 & 1155.13 \\
\hline
\end{tabular}

The DSOS algorithm selects nine features in the average state for all regression datasets. The elapsed time for PSO in which the best answer has been obtained was lowest for this algorithm. LCA, LA and FOA are algorithms which their functional are the same and proper than other algorithms. It is also obvious that LA has the best confidence interval of all alternative approaches. Except for FOA, which has an ER_P value of 0.003 , ER_P is identical for all algorithms to three decimal places. In the same way as CR_CI, CR_P and CR_TS for all regression datasets, the highest ER_TS value was achieved by WCC. WCC, LCA and LA achieved the maximum value of correlation (CR) for all regression datasets.

SEN, PRE, FPR, and ACC are the most important comparison criteria for classification problems. A summary of Table 5 is shown in Table 8 , which indicates that LCA obtains the best results in terms of FPR and ACC, and LA achieves the best result for SEN. WCC also acquires the best result for PRE on average.

In a comprehensive comparison, we evaluate the performance of all algorithms and methods on BSEHOCK dataset that is larger than others. Unlike previous experiments which are based on single objective (ACC) score; this one is based on multi objective score for wrapper methods. In Table 9 in which the best values of each column have been determined; the results are observable for SVM, ANN and DT learner. PCRR, LAP, ENT and MI are abbreviation for pearson correlation, laplacian, entropy and mutual information respectively in Table 9. As it is observed, every classifier and every feature selection method have their own attitude toward the data. Therefore, a user can apply various methods and algorithms along with different learners, and then can select the features which satisfy his/hers requirements. Also, it is possible that a user employee ensemble.

\section{Discussion}

Feature selection is one the most important steps in machine learning applications. For this purpose, many tools and methods have been introduced by researchers. For example, a feature weighting tool for unsupervised applications [54] and Weka machine learning tool [55] have been developed. However, the main limitation of these

Table 7 Summary of results for all regression datasets

\begin{tabular}{|c|c|c|c|c|c|c|c|c|c|c|c|c|c|c|}
\hline $\mathrm{AL}$ & NOF & ET & $E R$ & ER_STD & ER_Cl_1 & ER_Cl_2 & ER_P & ER_TS & $C R$ & CR_STD & CR_Cl_1 & CR_Cl_2 & CR_P & CR_TS \\
\hline$\overline{\text { WCC }}$ & 12.5 & 107.5 & 0.033 & 0.000 & 0.030 & 0.033 & 0.000 & $1.3 \mathrm{E}+15$ & 0.65 & 0.020 & 0.615 & 0.640 & 0.000 & $2.5 E+14$ \\
\hline LCA & 10.25 & 124 & 0.030 & 0.000 & 0.030 & 0.033 & 0.000 & 1274.13 & 0.65 & 0.005 & 0.610 & 0.633 & 0.000 & 916.1775 \\
\hline GA & 14.5 & 53 & 0.033 & 0.000 & 0.035 & 0.035 & 0.000 & 818.640 & 0.57 & 0.015 & 0.515 & 0.598 & 0.001 & 212.5 \\
\hline PSO & 14.00 & 50.5 & 0.033 & 0.000 & 0.033 & 0.033 & 0.000 & 435.880 & 0.56 & 0.045 & 0.520 & 0.583 & 0.001 & 225.3125 \\
\hline $\mathrm{ACO}$ & 14.00 & 128.25 & 0.033 & 0.000 & 0.035 & 0.035 & 0.000 & 290.915 & 0.57 & 0.050 & 0.473 & 0.570 & 0.003 & 125.4075 \\
\hline ICA & 13.50 & 53 & 0.033 & 0.000 & 0.035 & 0.035 & 0.000 & 813.673 & 0.60 & 0.005 & 0.578 & 0.588 & 0.000 & 488.6925 \\
\hline LA & 12.50 & 69 & 0.030 & 0.000 & 0.028 & 0.030 & 0.000 & 565.238 & 0.65 & 0.015 & 0.598 & 0.635 & 0.000 & 379.07 \\
\hline HTS & 12.00 & 70.5 & 0.033 & 0.000 & 0.035 & 0.038 & 0.000 & 406.563 & 0.57 & 0.043 & 0.518 & 0.573 & 0.001 & 145.995 \\
\hline FOA & 9.50 & 136.75 & 0.030 & 0.000 & 0.030 & 0.035 & 0.003 & 403.343 & 0.63 & 0.073 & 0.488 & 0.640 & 0.021 & 276.025 \\
\hline DSOS & 9.00 & 105.5 & 0.033 & 0.000 & 0.035 & 0.038 & 0.000 & 325.99 & 0.55 & 0.045 & 0.478 & 0.538 & 0.003 & 213.69 \\
\hline CUK & 10.50 & 124.5 & 0.033 & 0.000 & 0.033 & 0.033 & 0.000 & 998.68 & 0.60 & 0.010 & 0.555 & 0.590 & 0.001 & 662.7475 \\
\hline
\end{tabular}


Table 8 Summary of essential statistical criteria for all classification datasets

\begin{tabular}{lllll}
\hline AL_NAME & SEN & PRE & FPR & ACC \\
\hline WCC & 0.6800 & 0.7900 & 0.1525 & 0.8125 \\
LCA & 0.6900 & 0.7675 & 0.1450 & 0.8200 \\
GA & 0.6600 & 0.7475 & 0.1775 & 0.7525 \\
PSO & 0.6625 & 0.7475 & 0.1775 & 0.7600 \\
ACO & 0.6525 & 0.7425 & 0.1825 & 0.7475 \\
ICA & 0.6575 & 0.7550 & 0.1750 & 0.7625 \\
LA & 0.6925 & 0.7400 & 0.1500 & 0.8075 \\
HTS & 0.5825 & 0.6900 & 0.2125 & 0.6800 \\
FOA & 0.6475 & 0.7475 & 0.1775 & 0.7925 \\
DSOS & 0.6350 & 0.7300 & 0.1875 & 0.7375 \\
CUK & 0.6800 & 0.7275 & 0.1550 & 0.7950 \\
\hline
\end{tabular}

tools like mRMR [56] and mRMD [57] is that they are based on filter methods which only consider the relation among features and disregard interaction between feature selection algorithm and learner. As another example, we can mention a wrapper feature selection tool which is based on genetic algorithm [58]. Although time complexity of wrapper methods are higher than filter ones, these methods can lead better results; and it is valuable to spend more time. In this paper, we proposed a machine learning software named FeatureSelect that includes three types of popular learners (SVM, ANN and DT). In addition, two types of feature selection method are available in it. First method is wrapper method that is based on optimisation algorithms. Eleven state-of-art optimisation algorithms have been selected based on their popularity, novelty and functionality, and then implemented in FeatureSelect. Second type is the filter method which is based on Pearson correlation, entropy, Laplacian, mutual information and fisher scores. A user can also combine existing methods and algorithms, and then use them as ensemble or hybrid method like hybrid feature selection methods [59]. For example, a user can confine a number of features to specific threshold using filter methods. After it, the user can use wrapper methods along with an agile learner such as SVM or DT for acquiring an optimal subset of features, and finally engage and test ANN with enhancing a number of training iterations to obtain suitable model. There are also some other application-specific tools like iFeature [60] which is used for extracting and selecting features from protein and peptide sequences. Although iFeature includes a web server besides a stand-alone tool, FeatureSelect is the general software and provides different capabilities like hybrid feature selection and ensemble learning based on various states of combining filter and wrapper methods. In order to show capabilities of FeatureSelect, we applied it on various datasets with different sizes in multiple areas. The results show that every algorithm and every learner has its attitude relative to data, and algorithms' performances vary on different data. In another comprehensive experiment, we applied all of algorithms and learners of FeatureSelect on the BASEHOCK dataset with multi-objective score function. Although filter

Table 9 A comprehensive comparison of all methods

\begin{tabular}{|c|c|c|c|c|c|c|c|c|c|c|c|c|c|c|c|}
\hline \multirow[t]{2}{*}{$\mathrm{AL}$} & \multicolumn{5}{|c|}{ Learner $=$ SVM } & \multicolumn{5}{|c|}{ Learner $=$ ANN } & \multicolumn{5}{|c|}{ Learner = Decision tree } \\
\hline & SEN & SPC & PRE & FPR & ACC & SEN & SPC & PRE & FPR & ACC & SEN & SPC & PRE & FPR & $A C C$ \\
\hline WCC & 0/92 & $0 / 25$ & $0 / 43$ & $0 / 75$ & $0 / 51$ & 0/94 & $0 / 21$ & $0 / 63$ & 0/79 & $0 / 63$ & $0 / 45$ & $0 / 69$ & $0 / 34$ & $0 / 31$ & $0 / 52$ \\
\hline LCA & 0/92 & $0 / 25$ & $0 / 43$ & $0 / 75$ & $0 / 51$ & 0/85 & $0 / 24$ & 0/70 & 0/76 & $0 / 70$ & $0 / 46$ & $0 / 67$ & $0 / 36$ & $0 / 33$ & $0 / 50$ \\
\hline GA & 0/92 & $0 / 25$ & $0 / 43$ & $0 / 75$ & $0 / 51$ & 0/96 & 0/02 & $0 / 63$ & 0/98 & $0 / 63$ & $0 / 44$ & $0 / 61$ & $0 / 33$ & $0 / 39$ & $0 / 45$ \\
\hline PSO & 0/92 & $0 / 25$ & $0 / 43$ & $0 / 75$ & $0 / 51$ & $1 / 00$ & $0 / 00$ & $0 / 65$ & $1 / 00$ & $0 / 65$ & $0 / 44$ & $0 / 63$ & $0 / 31$ & $0 / 37$ & $0 / 47$ \\
\hline $\mathrm{ACO}$ & 0/92 & $0 / 25$ & $0 / 43$ & $0 / 75$ & $0 / 51$ & 0/97 & $0 / 14$ & $0 / 72$ & $0 / 86$ & $0 / 72$ & $0 / 43$ & $0 / 60$ & $0 / 31$ & $0 / 40$ & $0 / 43$ \\
\hline ICA & 0/92 & $0 / 25$ & $0 / 43$ & $0 / 75$ & $0 / 51$ & $1 / 00$ & 0/00 & $0 / 70$ & $1 / 00$ & $0 / 70$ & $0 / 44$ & $0 / 62$ & $0 / 33$ & $0 / 38$ & $0 / 45$ \\
\hline LA & 0/92 & $0 / 25$ & $0 / 43$ & $0 / 75$ & $0 / 51$ & $1 / 00$ & 0/00 & $0 / 73$ & $1 / 00$ & $0 / 73$ & $0 / 45$ & $0 / 63$ & $0 / 36$ & $0 / 37$ & $0 / 42$ \\
\hline HTS & 0/93 & $0 / 21$ & $0 / 42$ & 0/79 & $0 / 49$ & 0/90 & $0 / 33$ & $0 / 55$ & $0 / 67$ & $0 / 55$ & $0 / 43$ & $0 / 57$ & $0 / 31$ & $0 / 43$ & $0 / 41$ \\
\hline FOA & 0/90 & $0 / 32$ & $0 / 46$ & $0 / 68$ & $0 / 54$ & 0/94 & $0 / 22$ & $0 / 67$ & 0/78 & $0 / 67$ & $0 / 44$ & $0 / 63$ & $0 / 34$ & $0 / 37$ & $0 / 46$ \\
\hline DSOS & 0/92 & $0 / 25$ & $0 / 43$ & $0 / 75$ & $0 / 51$ & $0 / 74$ & $0 / 51$ & $0 / 67$ & $0 / 49$ & $0 / 67$ & $0 / 44$ & $0 / 61$ & $0 / 34$ & 0/39 & $0 / 44$ \\
\hline CUK & 0/92 & $0 / 25$ & $0 / 43$ & $0 / 75$ & $0 / 51$ & $0 / 83$ & $0 / 40$ & $0 / 65$ & $0 / 60$ & $0 / 65$ & $0 / 43$ & $0 / 59$ & $0 / 28$ & $0 / 41$ & $0 / 43$ \\
\hline PCRR & $0 / 98$ & 0/04 & $0 / 36$ & 0/96 & $0 / 43$ & 0/96 & 0/02 & $0 / 67$ & 0/98 & $0 / 67$ & $0 / 43$ & $0 / 28$ & $0 / 15$ & $0 / 72$ & $0 / 17$ \\
\hline LAP & 0/94 & $0 / 17$ & $0 / 40$ & 0/83 & $0 / 48$ & 0/77 & $0 / 35$ & $0 / 67$ & $0 / 65$ & $0 / 67$ & $0 / 44$ & 0/39 & $0 / 18$ & $0 / 61$ & $0 / 27$ \\
\hline ENT & 0/94 & $0 / 17$ & $0 / 40$ & 0/83 & $0 / 48$ & $1 / 00$ & 0/00 & $0 / 67$ & 1 & $0 / 67$ & $0 / 43$ & $0 / 61$ & $0 / 30$ & 0/39 & $0 / 45$ \\
\hline $\mathrm{MI}$ & $1 / 00$ & 0/00 & $0 / 35$ & $1 / 00$ & $0 / 41$ & $1 / 00$ & 0/00 & $0 / 68$ & 1 & $0 / 68$ & $0 / 50$ & 0/00 & 0/00 & $1 / 00$ & $0 / 00$ \\
\hline Fisher & $1 / 00$ & 0/00 & $0 / 35$ & $1 / 00$ & $0 / 41$ & 0/98 & 0/06 & $0 / 67$ & 0/94 & $0 / 67$ & $0 / 50$ & $0 / 00$ & $0 / 00$ & $1 / 00$ & $0 / 00$ \\
\hline
\end{tabular}

Boldface values indicate the best-obtained results of each criterion for every learner 
methods are quicker than wrapper methods, the acquired results present that wrapper methods' performance are proper than the filter methods.

\section{Conclusions}

In this paper, a new software application for feature selection is proposed. This software is called FeatureSelect, and can be used in fields such as biology, image processing, drug design and numerous other domains. FeatureSelect selects a subset of features using optimisation algorithms with considering different score functions and then transmits these to the learner. SVM, ANN and DT are used here as a learner that can be applied to classification and regression datasets. Since LIBSVM is a library for SVM and provides a wide range of options for classification and regression problems, we developed FeatureSelect based on this library. Researchers can apply FeatureSelect to any dataset using three types of learners and two types of feature selection methods and obtain various tables and diagrams based on the nature of the dataset. It is also possible to combine the methods and algorithms as ensemble method. FeatureSelect was applied to eight datasets with differing scope and size. We then compared the performance of the algorithms in FeatureSelect to these datasets and presented some examples of the outputs in the form of tables and diagrams. Although the algorithms and feature selection methods have different functionality for different datasets, WCC, LCA, LA and FOA are the algorithms having proper functionality than others, and wrapper methods lead better results than filter methods.

\section{Additional file}

Additional file 1: The supplementary file. It consists of source codes FeatureSelect has been implemented in MATLAB and is free open source software. Therefore, users can change or improve it. The modified versions of it will be uploaded to the GltHub repository. Also, three types of stand-alone versions of FeatureSelect, including WIN 64-bit, java, and python packages, are available. (ZIP $151 \mathrm{mb}$ )

\section{Abbreviations}

ACC: Accuracy; ACO: Ant Colony Optimization; ANN: Artificial Neural Network; CUK: Cuckoo algorithm; DSOS: Discrete Symbiotic Optimization Search; ER: Error; FOA: Forest Optimization Algorithm; FPR: False Positive Rate; FS: Feature Selection; GA: Genetic Algorithm; HTS: Heat Transfer Optimization; ICA: Imperialist Competitive Algorithm; LA: Learning Automata; LCA: League Championship Algorithm; PRE: Precision; PSO: Particle Swarm Optimization; SEN: Sensitivity; SPC: Specificity; SVM: Support Vector Machine; WCC: World Competitive Contest Algorithm

\section{Acknowledgements}

Not applicable.

\section{Availability and requirements}

Project name: FeatureSelect. Project homepage: https://github.com/LBBSoft/ FeatureSelect, Operating systems: Win 10, Linux, and Mac. Programing language: MATLAB. Requirements: MATLAB Runtime, SDK, python 2.7, 3.4, or 3.5 (if a user runs the FeatureSelect using the python package), and java version 1.8 (if a user runs the FeatureSelect using the java package). License: MIT. Any restrictions to use by non-academics: MIT license.

\section{Funding}

No funding.

\section{Availability of data and materials}

FeatureSelect has been implemented in MATLAB programing language and is available at (https://github.com/LBBSoft/FeatureSelect). In addition to the code and datasets, three stand-alone versions including java-package, python package, and an exe file for win_64_bit are also accessible.

\section{Authors' contributions}

YMS: Conceptualization, software programming, formal analysis, investigation, writing-manuscript. HMG: Software testing, validation, visualization writingmanuscript. AMN: Conceptualization, Supervision, Project administration, Editing the manuscript. All authors have read and approved the manuscript.

Ethics approval and consent to participate

Not applicable.

Consent for publication

Not applicable.

\section{Competing interests}

The authors declare that they have no competing interests.

\section{Publisher's Note}

Springer Nature remains neutral with regard to jurisdictional claims in published maps and institutional affiliations.

Received: 6 December 2018 Accepted: 19 March 2019

Published online: 03 April 2019

\section{References}

1. Miao J, Niu L. A survey on feature selection. Procedia Computer Science. 2016;91:919-26.

2. MotieGhader $\mathrm{H}$, Gharaghani $\mathrm{S}$, Masoudi-Sobhanzadeh $\mathrm{Y}$, Masoudi-Nejad A. Sequential and mixed genetic algorithm and learning automata (SGALA, MGALA) for feature selection in QSAR. Iranian Journal of Pharmaceutical Research. 2017;16(2):533-53.

3. Sheikhpour R, Sarram MA, Gharaghani S, Chahooki MAZ. A survey on semi-supervised feature selection methods. Pattern Recogn. 2017;64: 141-58.

4. Ghaddar B, Naoum-Sawaya J. High dimensional data classification and feature selection using support vector machines. Eur J Oper Res. 2017

5. Liu B, Liu F, Wang X, Chen J, Fang L, Chou K-C. Pse-in-one: a web server for generating various modes of pseudo components of DNA, RNA, and protein sequences. Nucleic Acids Res. 2015;43(W1):W65-71.

6. Xiao N, Cao D-S, Zhu M-F, Xu Q-S. Protr/ProtrWeb: R package and web server for generating various numerical representation schemes of protein sequences. Bioinformatics. 2015;31(11):1857-9.

7. Rahmaninia M, Moradi P. OSFSMI: online stream feature selection method based on mutual information. Appl Soft Comput. 2017.

8. Che J, Yang Y, Li L, Bai X, Zhang S, Deng C. Maximum relevance minimum common redundancy feature selection for nonlinear data. Inf Sci. 2017;409:68-86.

9. Sanz H, Valim C, Vegas E, Oller JM, Reverter F. SVM-RFE: selection and visualization of the most relevant features through non-linear kernels. BMC bioinformatics. 2018;19(1):432.

10. Viegas F, Rocha L, Gonçalves M, Mourão F, Sá G, Salles T, Andrade G, Sandin I. A genetic programming approach for feature selection in highly dimensional skewed data. Neurocomputing. 2017.

11. Izetta J, Verdes PF, Granitto PM. Improved multiclass feature selection via list combination. Expert Syst Appl. 2017;88:205-16.

12. Xiao J, Cao H, Jiang X, Gu X, Xie L. GMDH-based semi-supervised feature selection for customer classification. Knowl-Based Syst. 2017.

13. Liu J, Lin Y, Lin M, Wu S, Zhang J. Feature selection based on quality of information. Neurocomputing. 2017;225:11-22.

14. Goswami S, Das AK, Chakrabarti A, Chakraborty B. A feature cluster taxonomy based feature selection technique. Expert Syst Appl. 2017;79:76-89.

15. Zhou P, Hu X, Li P, Wu X. Online feature selection for high-dimensional class-imbalanced data. Knowl-Based Syst. 2017.

16. Yu K, Ding W, Wu X. LOFS: a library of online streaming feature selection. Knowl-Based Syst. 2016;113:1-3. 
17. Wu Y, Liu Y, Wang Y, Shi Y, Zhao X. JCDSA: a joint covariate detection tool for survival analysis on tumor expression profiles. BMC bioinformatics. 2018; 19(1):187.

18. Yang R, Zhang C, Zhang L, Gao R. A two-step feature selection method to predict Cancerlectins by Multiview features and synthetic minority oversampling technique. Biomed Res Int. 2018;2018.

19. Ge R, Zhou M, Luo Y, Meng Q, Mai G, Ma D, Wang G, Zhou F. McTwo: a two-step feature selection algorithm based on maximal information coefficient. BMC bioinformatics. 2016;17(1):142.

20. Metin SK. Feature selection in multiword expression recognition. Expert Syst Appl. 2017.

21. Lu H, Chen J, Yan K, Jin Q, Xue Y, Gao Z. A hybrid feature selection algorithm for gene expression data classification. Neurocomputing. 2017.

22. Maldonado S, Lopez J. Synchronized feature selection for support vector machines with twin hyperplanes. Knowl-Based Syst. 2017:132:119-28.

23. Ma B, Xia Y. A tribe competition-based genetic algorithm for feature selection in pattern classification. Appl Soft Comput. 2017:58:328-38

24. Peng $H$, Fan Y: Feature selection by optimizing a lower bound of conditional mutual information. Information Sciences 2017, 418(Supplement C):652-667.

25. Hamedmoghadam-Rafati H, Jalili M, Yu X. An opinion formation based binary optimization approach for feature selection. Physica A: Statistical Mechanics and its Applications. 2017.

26. Chandrashekar G, Sahin F. A survey on feature selection methods. Computers \& Electrical Engineering. 2014;40(1):16-28.

27. Lazar C, Taminau J, Meganck S, Steenhoff D, Coletta A, Molter C, de Schaetzen $V$, Duque R, Bersini H, Nowe A. A survey on filter techniques for feature selection in gene expression microarray analysis. IEEE/ACM Transactions on Computational Biology and Bioinformatics (TCBB). 2012;9(4):1106-19.

28. Lee PY, Loh WP, Chin JF. Feature selection in multimedia: the state-of-theart review. Image Vis Comput. 2017.

29. Panday D, Cordeiro de Amorim R, Lane P. Feature weighting as a tool for unsupervised feature selection. Inf Process Lett. 2017.

30. Sadeghianpourhamami N, Ruyssinck J, Deschrijver D, Dhaene T, Develder C. Comprehensive feature selection for appliance classification in NILM. Energy and Buildings. 2017;151:98-106.

31. Du S, Ma Y, Li S, Ma Y. Robust unsupervised feature selection via matrix factorization. Neurocomputing. 2017;241:115-27.

32. Agnihotri D, Verma K, Tripathi P. Variable global feature selection scheme for automatic classification of text documents. Expert Syst Appl. 2017;81: 268-81.

33. Oreski D, Oreski S, Klicek B. Effects of dataset characteristics on the performance of feature selection techniques. Appl Soft Comput. 2017;52:109-19.

34. Liu M, Zhang D. Feature selection with effective distance. Neurocomputing. 2016;215:100-9.

35. Das AK, Goswami S, Chakrabarti A, Chakraborty B. A new hybrid feature selection approach using feature association map for supervised and unsupervised classification. Expert Syst Appl. 2017:88:81-94.

36. He W, Cheng X, Hu R, Zhu Y, Wen G. Feature self-representation based hypergraph unsupervised feature selection via low-rank representation. Neurocomputing. 2017;253:127-34.

37. Liu H, Yu L. Toward integrating feature selection algorithms for classification and clustering. IEEE Trans Knowl Data Eng. 2005;17(4):491-502.

38. Liang D, Tsai C-F, Wu H-T. The effect of feature selection on financial distress prediction. Knowl-Based Syst. 2015;73:289-97.

39. Golay J, Leuenberger $M$, Kanevski M. Feature selection for regression problems based on the Morisita estimator of intrinsic dimension. Pattern Recogn. 2017;70:126-38.

40. Yu S, Zhao H. Rough sets and Laplacian score based cost-sensitive feature selection. PLoS One. 2018;13(6):e0197564.

41. Jiang F, Sui Y, Zhou L. A relative decision entropy-based feature selection approach. Pattern Recogn. 2015;48(7):2151-63.

42. Gu Q, Li Z, Han J: Generalized fisher score for feature selection. arXiv preprint arXiv:12023725 2012

43. Hira ZM, Gillies DF. A review of feature selection and feature extraction methods applied on microarray data. Adv Bioinforma. 2015;2015.

44. Hancer $E$, Xue B, Zhang M. Differential evolution for filter feature selection based on information theory and feature ranking. Knowl-Based Syst. 2018; 140:103-19.

45. Cortes C, Vapnik V. Support-vector networks. Mach Learn. 1995;20(3):273-97.

46. Ben-Hur A, Horn D, Siegelmann HT, Vapnik V. Support vector clustering. J Mach Learn Res. 2001;2(Dec):125-37.
47. Chang C-C, Lin C-J. LIBSVM: a library for support vector machines. ACM transactions on intelligent systems and technology (TIST). 2011;2(3):27.

48. Li Y, Wei B, Liu Y, Yao L, Chen H, Yu J, Zhu W. Incorporating knowledge into neural network for text representation. Expert Syst Appl. 2018;96:103-14.

49. Wang L, Li Q, Yu Y, Liu J. Region compatibility based stability assessment for decision trees. Expert Syst Appl. 2018;105:112-28.

50. Diaz-Hermida F, Pereira-Fariña M, Vidal JC, Ramos-Soto A. Characterizing quantifier Fuzzification mechanisms: a behavioral guide for applications. Fuzzy Sets Syst. 2017.

51. Črepinšek M, Liu S-H, Mernik M. Replication and comparison of computational experiments in applied evolutionary computing: common pitfalls and guidelines to avoid them. Appl Soft Comput. 2014;19:161-70.

52. Schubert A-L, Hagemann D, Voss A, Bergmann K: Evaluating the model fit of diffusion models with the root mean square error of approximation. Journal of Mathematical Psychology 2017, 77(Supplement C):29-45.

53. Hanley JA, MCNeil BJ. The meaning and use of the area under a receiver operating characteristic (ROC) curve. Radiology. 1982;143(1):29-36.

54. Panday D, de Amorim RC, Lane P. Feature weighting as a tool for unsupervised feature selection. Inf Process Lett. 2018;129:44-52.

55. Witten $I H$, Frank E, Trigg LE, Hall MA, Holmes G, Cunningham SJ. Weka: practical machine learning tools and techniques with Java implementations; 1999.

56. $\quad$ Ding $\mathrm{C}$, Peng $\mathrm{H}$. Minimum redundancy feature selection from microarray gene expression data. J Bioinforma Comput Biol. 2005;3(02):185-205.

57. Wei L, Xing P, Shi G, Ji Z-L, Zou Q. Fast prediction of protein methylation sites using a sequence-based feature selection technique. IEEE/ACM Transactions on Computational Biology and Bioinformatics. 2017;1:1-1.

58. Soufan O, Kleftogiannis D, Kalnis P, Bajic VB. DWFS: a wrapper feature selection tool based on a parallel genetic algorithm. PLoS One. 2015;10(2):e0117988.

59. Wang $Y$, Feng L. Hybrid feature selection using component co-occurrence based feature relevance measurement. Expert Syst Appl. 2018;102:83-99.

60. Chen Z, Zhao P, Li F, Leier A, Marquez-Lago T, Wang Y, Webb Gl, Smith Al, Daly RJ, Chou K-C. iFeature: a python package and web server for features extraction and selection from protein and peptide sequences. Bioinformatics. 2018;1:4.

61. Masoudi-Sobhanzadeh Y, Motieghader H: World Competitive Contests (WCC) algorithm: A novel intelligent optimization algorithm for biological and non-biological problems. Informatics in Medicine Unlocked 2016, 3(Supplement C):15-28.

62. Husseinzadeh Kashan A: League Championship Algorithm (LCA): An algorithm for global optimization inspired by sport championships. Applied Soft Computing 2014, 16(Supplement C):171-200.

63. Holland $\mathrm{JH}$. Searching nonlinear functions for high values. Appl Math Comput. 1989;32(2):255-74.

64. Eberhart R, Kennedy J: A new optimizer using particle swarm theory. In: Micro Machine and Human Science, 1995 MHS'95, Proceedings of the Sixth International Symposium on: 1995. IEEE: 39-43.

65. Dorigo M, Birattari M, Stutzle T. Ant colony optimization. IEEE Comput Intell Mag. 2006:1(4):28-39.

66. Atashpaz-Gargari E, Lucas C: Imperialist competitive algorithm: an algorithm for optimization inspired by imperialistic competition. In: Evolutionary computation, 2007 CEC 2007 IEEE congress on: 2007. IEEE: 4661-4667.

67. Meybodi MR, Beigy H. New learning automata based algorithms for adaptation of backpropagation algorithm parameters. Int J Neural Syst. 2002;12(01):45-67.

68. Patel VK, Savsani VJ: Heat transfer search (HTS): a novel optimization algorithm. Information Sciences 2015, 324(Supplement C):217-246.

69. Ghaemi M, Feizi-Derakhshi M-R. Forest optimization algorithm. Expert Syst Appl. 2014;41(15):6676-87.

70. Ezugwu AE-S, Adewumi AO: Discrete symbiotic organisms search algorithm for travelling salesman problem. Expert Systems with Applications 2017 87(Supplement C):70-78.

71. Rajabioun R. Cuckoo optimization algorithm. Appl Soft Comput. 2011;11(8): 5508-18.

72. Fernandes $K$, Vinagre $P$, Cortez $P$ : A proactive intelligent decision support system for predicting the popularity of online news. In: Portuquese Conference on Artificial Intelligence: 2015. Springer: 535-546.

73. Laufer R, Ng G, Liu Y, Patel NKB, Edwards LG, Lang Y, Li S-W, Feher M, Awrey DE, Leung G. Discovery of inhibitors of the mitotic kinase TTK based on N-(3-(3-sulfamoylphenyl)-1H-indazol-5-yl)-acetamides and carboxamides. Bioorg Med Chem. 2014;22(17):4968-97. 
74. De Vito S, Massera E, Piga M, Martinotto L, Di Francia G. On field calibration of an electronic nose for benzene estimation in an urban pollution monitoring scenario. Sensors Actuators B Chem. 2008;129(2):750-7.

75. Candanedo LM, Feldheim V, Deramaix D. Data driven prediction models of energy use of appliances in a low-energy house. Energy and Buildings. 2017;140:81-97.

76. Li J, Cheng K, Wang S, Morstatter F, Trevino RP, Tang J, Liu H: Feature selection: A data perspective. arXiv preprint arXiv:160107996 2016.

77. Diaz-Chito K, Hernández-Sabaté A, López AM. A reduced feature set for driver head pose estimation. Appl Soft Comput. 2016;45:98-107.

Ready to submit your research? Choose BMC and benefit from:

- fast, convenient online submission

- thorough peer review by experienced researchers in your field

- rapid publication on acceptance

- support for research data, including large and complex data types

- gold Open Access which fosters wider collaboration and increased citations

- maximum visibility for your research: over $100 \mathrm{M}$ website views per year

At BMC, research is always in progress.

Learn more biomedcentral.com/submissions 